\title{
Approximate $\kappa$-state solutions to the Dirac-Yukawa problem based on the spin and pseudospin symmetry
}

\author{
Sameer M. Ikhdair $1, *$ \\ ${ }^{1}$ Physics Department, Near East University, Nicosia, North Cyprus, Turkey
}

(Dated: November 24, 2018)

\begin{abstract}
Using an approximation scheme to deal with the centrifugal (pseudo-centrifugal) term, we solve the Dirac equation with the screened Coulomb (Yukawa) potential for any arbitrary spin-orbit quantum number $\kappa$. Based on the spin and pseudospin symmetry, analytic bound state energy spectrum formulas and their corresponding upper- and lower-spinor components of two Dirac particles are obtained using a shortcut of the Nikiforov-Uvarov method. We find a wide range of permissible values for the spin symmetry constant $C_{s}$ from the valence energy spectrum of particle and also for pseudospin symmetry constant $C_{p s}$ from the hole energy spectrum of antiparticle. Further, we show that the present potential interaction becomes less (more) attractive for a long (short) range screening parameter $\alpha$. To remove the degeneracies in energy levels we consider the spin and pseudospin solution of Dirac equation for Yukawa potential plus a centrifugal-like term. A few special cases such as the exact spin (pseudospin) symmetry Dirac-Yukawa, the Yukawa plus centrifugal-like potentials, the limit when $\alpha$ becomes zero (Coulomb potential field) and the nonrelativistic limit of our solution are studied. The nonrelativistic solutions are compared with those obtained by other methods.

Keywords: Dirac equation, spin symmetry, pseudospin symmetry, screened Coulomb potential, approximation scheme; Nikiforov-Uvarov method

PACS numbers: 03.65.Ge; 03.65.Db; 03.65.Pm; 21.45.Bc
\end{abstract}

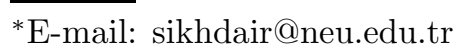




\section{INTRODUCTION}

The screened Coulomb (Yukawa) potential is widely used in physics, being a good approximation to short-range interactions between charged particles in various areas of physics $[1,2]$. In plasma physics it is known as the Debye-Hückel potential describes the shielding effect of ions embedded in plasmas [3]. It has also been used to play a fundamental role in (dusty/complex) plasma and colloidal suspensions. The momentum transfer in pair collisions of particles interacting via the Yukawa potential is well investigated in the limit when the interaction is "weak" in the sense that its range (distance at which the interaction energy is equal to the kinetic energy) is much shorter than the plasma screening length. This limit is known as the theory of Coulomb scattering and is extensively used to describe collisions in usual electron-ion plasma [4]. In solid state, atomic and molecular physics it is called the Thomas-Fermi or screened Coulomb potential due to the cloud of electronic charges around the nucleus $[5,6]$. Also this potential is well known in nuclear physics as the dominant central part of neutrons-protons nuclear interaction due to the massive field exchange (one pion) whose mass is $m[7,8]$. In high energy physics, the potential is used to model the interaction of hadrons in short range gauge theories where coupling is mediated by the exchange of a massive scalar meson $[1,9]$. It is defined as follows [10]:

$$
V(r)=-\frac{A}{r} e^{-\alpha r}, A>0
$$

where $\alpha$ and $A$ are the screening (range) and coupling strength parameters, respectively. The two parameters are given by different expressions depending on the type of the problem under consideration. For example, $A=g^{2}$ is positive for attraction, $g$ denotes the coupling constant between meson field and the fermion field with which it interacts. Since the field mediator is massive, the corresponding force has a certain range, which is inversely proportional to the mass, $\alpha=m c / \hbar$ If the mass is zero, then the Yukawa potential becomes equal to a Coulomb potential and the range is said to be infinite. Further, the number of bound states of the Yukawa potential is found to be finite. Unfortunately, since the Schrödinger equation for the screened Coulomb potential does not admit an exact analytical solution [11], therefore, various numerical [12,13] and analytical [14-17] methods have been developed in the past. Also the energy spectrum can be calculated with high accuracy by means of the

hypervirial relations and Pade approximation methods $[18,19]$. The short-range behavior of the decaying exponential factor $e^{-\alpha r}$ and singularity at $r=0$ make the task of obtaining 
accurate solutions a difficult task. Besides, most of these calculations suffer from limited accuracy when a wider range of potential parameters are being considered [20].

An approximate perturbative method has been developed to obtain the energy spectrum and wave functions of the Schrödinger with the Yukawa-like potentials [21]. This method has been applied to obtain the energy spectrum and wave functions for the more general exponential-cosine-screened Coulomb potentials. These potentials are containing an additional Coulomb term superposed with the Yukawa potential that might be useful in describing the effective interaction in many-body problem [22]. Further, the asymptotic iteration method is used to obtain the energy eigenvalues of the Yukawa potential [23]. The $J$-matrix method has been applied to the Yukawa potential with no special treatment of its singularity by using the oscillator basis and the reference Hamiltonian contained only the kinetic energy operator [24]. The bound states spectrum and resonance energies of the Yukawa potential have been studied using the method of complex scaling [25]. Therefore, an alternative Laguerre basis has also been used to comply with the $J$-matrix requirement of a tridiagonal matrix representation of the reference Hamiltonian that includes the $r^{-1}$ singularity [18].

When a particle is in a strong interaction (range of interaction exceeds the screening length, $\lambda=\alpha^{-1}$ ), the relativistic effect must be considered which gives the correction for nonrelativistic quantum mechanics. The relativistic treatment is of much interest especially when (at least) one of the particles is highly charged and their relative velocity is small. Taking the relativistic effects into account, a particle in a potential field should be described with the Dirac equation. Therefore, the solution of the Dirac equation can be important in different fields of physics like nuclear and molecular physics $[7,26]$. Within the framework of the Dirac equation the spin symmetry arises if the magnitude of the spherical attractive scalar potential $S(r)$ and repulsive vector potential are nearly equal (i.e., $S(r) \sim V(r)$ ) in the nuclei (i.e., when the difference potential $\Delta(r)=V(r)-S(r)=C_{s}$, with $C_{s}$ is an arbitrary constant); however, the pseudospin symmetry occurs if $S(r) \sim-V(r)$ are nearly equal (i.e., when the sum potential $\Sigma(r)=V(r)+S(r)=C_{p s}$, with $C_{p s}$ is an arbitrary constant) [27]. The spin symmetry is relevant for mesons [28]. The pseudospin symmetry concept has been applied to many systems in nuclear physics and related areas [27-31] and used to explain features of deformed nuclei [32], the super-deformation [33] and to establish an effective nuclear shell-model scheme $[29,30,34]$. Recently, the spin and pseudospin symmetries have 
been widely applied on several physical potentials by many authors (cf. [35-37] and references therein). Many authors have investigated approximately the solution of the Dirac equation with a few potential models such as the the generalized Morse potential [35], the Hulthén potential [36], the Rosen-Morse potential [37] and the screened Coulomb potential [38] etc within the framework of various methods.

In the framework of the spin symmetry $S(r) \sim V(r)$ and pseudospin symmetry $S(r) \sim$ $-V(r)$, the bound state energy eigenvalues and associated upper- and lower-spinor wave functions are investigated by means of the Nikiforov-Uvarov (NU) method [39]. We have approximately solved the Dirac equation for the Hulthen potential [36] with spin and pseudospin symmetry for any spin-orbit $\kappa$ state and found the eigenvalue equation and corresponding two-component spinors within the framework of an approximation to the term proportional to $1 / r^{2}$. We have also solved the $(3+1)$ dimensional Dirac equation for a single particle trapped in the spherically symmetric generalized WS potential under the conditions of exact spin and pseudospin symmetry combined with approximation for the spin-orbit centrifugal (pseudo-centrifugal) term, and calculated the two-component spinor wave functions and the energy eigenvalues for any arbitrary spin-orbit $\kappa$ bound states [40]. Recently, Setare and Haidari [41] have solved the Dirac-Yukawa problem in the presence of the spin symmetry and given only analytical expressions for energy eigenvalues and wave functions. However, they have not given further numerical discussions for the validity of their analytical solutions. On the other hand, the subject of the pseudospin symmetry of the Dirac-Yukawa problem introduced by Ginocchio [27] has not been investigated by Ref. [41]. Over the past years, the interest in the quality of the pseudospin symmetry has been increased in the framework of the single-particle relativistic potential models. Therefore, we have found that it is necessary to give a detailed study for the solution of the Dirac equation with screened Coulomb (Yukawa) potential model in the presence of spin as well as pseudospin symmetry in a very simple and elegant way by using a shortcut procedures for the NU method. We also give a detailed discussion for the validity of the present numerical as well as analytical solutions. We also try to explore the exact relativistic energy spectrum of the Coulombic field (when $\alpha=0$, the low screening range of the Yukawa potential) under the exact spin and pseudospin symmetry.

The analytic solution of the Dirac equation with the screened Coulomb potential is difficult to find due to the centrifugal (pseudo centrifugal) term $\kappa(\kappa+1) r^{-2}\left(\kappa(\kappa-1) r^{-2}\right)$ and 
the singular interactions like $r^{-1}$ (e.g., the Coulomb potential). Nevertheless, employing the approximation provided by Greene and Aldrich [42] to the centrifugal term $r^{-2}$ and to the

singular Coulombic part $r^{-1}$ makes the solution handy. We work within the framework of the low screening parameter throughout the paper. We find analytically approximate bound state solutions including the energy spectra and the corresponding spinor wave functions in the presence of the spin symmetry and pseudospin symmetry concept for any $\kappa$-state within the parametric generalization of the NU method [43] given in Appendix A

This paper is organized as follows. In section 2, we investigate the bound state energy equation and the corresponding two-component spinor wave functions in the presence of spin and pseudospin symmetry concept for the screened Coulomb potential by employing a parametric generalization of the NU method. In section 3, we study some special cases like Schrödinger-Yukawa, Dirac-Coulomb, exact spin (pseudospin) symmetric Dirac-Yukawa problem and Yukawa plus centrifugal-like potentials. In section 4, we present some numerical results to the non-relativistic and relativistic numerical energy levels for the Yukawa potential. The relevant conclusions are given in section 5 .

\section{THEORETICAL FRAMEWORK OF DIRAC BOUND STATE SOLUTIONS}

The Dirac equation for fermionic massive spin-1/2 particles moving in an attractive scalar potential $S(r)$ and a repulsive vector potential $V(r)$ is given by [7]

$$
\left[c \boldsymbol{\alpha} \cdot \mathbf{p}+\boldsymbol{\beta}\left(M c^{2}+S(r)\right)+V(r)-E\right] \psi_{n \kappa}(\mathbf{r})=0, \psi_{n \kappa}(\mathbf{r})=\psi(r, \theta, \phi),
$$

where $E$ is the relativistic energy of the system, $M$ is the mass of a particle, $\mathbf{p}=-i \hbar \boldsymbol{\nabla}$ is the momentum operator, and $\boldsymbol{\alpha}$ and $\boldsymbol{\beta}$ are $4 \times 4$ Dirac matrices, i.e.,

$$
\boldsymbol{\alpha}=\left(\begin{array}{cc}
0 & \boldsymbol{\sigma}_{i} \\
\boldsymbol{\sigma}_{i} & 0
\end{array}\right), \boldsymbol{\beta}=\left(\begin{array}{cc}
\mathbf{I} & 0 \\
0 & -\mathbf{I}
\end{array}\right), \sigma_{1}=\left(\begin{array}{cc}
0 & 1 \\
1 & 0
\end{array}\right), \sigma_{2}=\left(\begin{array}{cc}
0 & -i \\
i & 0
\end{array}\right), \sigma_{3}=\left(\begin{array}{cc}
1 & 0 \\
0 & -1
\end{array}\right)
$$

where $\mathbf{I}$ denotes the $2 \times 2$ identity matrix and $\boldsymbol{\sigma}_{i}$ are the three-vector Pauli spin matrices. In a spherical symmetrical nuclei, the total angular momentum operator of the nuclei $\mathbf{J}$ and spin-orbit matrix operator $\mathbf{K}=-\boldsymbol{\beta}(\boldsymbol{\sigma} \cdot \mathbf{L}+\mathbf{I})$ commute with the Dirac Hamiltonian, where $\mathbf{L}$ is the orbital angular momentum operator. The spinor wave functions can be classified according to the radial quantum number $n$ and the spin-orbit quantum number $\kappa$ and can 
be written using the Pauli-Dirac representation in the following forms:

$$
\psi_{n \kappa}(\mathbf{r})=\left(\begin{array}{c}
f_{n \kappa}(\mathbf{r}) \\
g_{n \kappa}(\mathbf{r})
\end{array}\right)=\frac{1}{r}\left(\begin{array}{c}
F_{n \kappa}(r) Y_{j m \kappa}^{l}(\widehat{r}) \\
i G_{n \kappa}(r) Y_{j m(-\kappa)}^{\widetilde{l}}(\widehat{r})
\end{array}\right)
$$

where the upper- and lower-spinor components $F_{n \kappa}(r)$ and $G_{n \kappa}(r)$ are the real square-integral radial wave functions, $Y_{j m \kappa}^{l}(\widehat{r})$ and $Y_{j m(-\kappa)}^{\widetilde{l}}(\widehat{r})$ are the spin spherical harmonic functions coupled to the total angular momentum $j$ and it's projection $m$ on the $z$ axis and $\kappa(\kappa+1)=$ $l(l+1)$ and $\kappa(\kappa-1)=\tilde{l}(\tilde{l}+1)$. The quantum number $\kappa$ is related to the quantum numbers for spin symmetry $l$ and pseudospin symmetry $\tilde{l}$ as

$$
\kappa=\left\{\begin{array}{ccc}
-(l+1)=-\left(j+\frac{1}{2}\right), & \left(s_{1 / 2}, p_{3 / 2}, \text { etc. }\right), & j=l+\frac{1}{2}, \quad \text { aligned spin }(\kappa<0), \\
+l=+\left(j+\frac{1}{2}\right), & \left(p_{1 / 2}, d_{3 / 2}, \text { etc. }\right), & j=l-\frac{1}{2}, \quad \text { unaligned } \operatorname{spin}(\kappa>0),
\end{array}\right.
$$

and the quasi-degenerate doublet structure can be expressed in terms of a pseudospin angular momentum $\widetilde{s}=1 / 2$ and pseudo-orbital angular momentum $\widetilde{l}$ which is defined as

$$
\kappa=\left\{\begin{array}{ccc}
-\widetilde{l}=-\left(j+\frac{1}{2}\right), & \left(s_{1 / 2}, \quad p_{3 / 2}, \quad \text { etc. }\right), \quad j=\widetilde{l}-1 / 2, \quad \text { aligned } \operatorname{spin}(\kappa<0), \\
+(\widetilde{l}+1)=+\left(j+\frac{1}{2}\right), & \left(d_{3 / 2}, \quad f_{5 / 2}, \quad \text { etc. }\right), \quad j=\widetilde{l}+1 / 2, \quad \text { unaligned } \operatorname{spin}(\kappa>0),
\end{array}\right.
$$

where $\kappa= \pm 1, \pm 2, \cdots$. For example, $\left(1 s_{1 / 2}, 0 d_{3 / 2}\right)$ and $\left(2 \mathrm{p}_{3 / 2}, 1 f_{5 / 2}\right)$ can be considered as pseudospin doublets.

Upon direct substitution of Eq. (4) into Eq. (2), the two radial coupled Dirac equations for the two spinor components can be obtained as

$$
\begin{aligned}
& \left(\frac{d}{d r}+\frac{\kappa}{r}\right) F_{n \kappa}(r)=\left[M c^{2}+E_{n \kappa}-\Delta(r)\right] G_{n \kappa}(r), \\
& \left(\frac{d}{d r}-\frac{\kappa}{r}\right) G_{n \kappa}(r)=\left[M c^{2}-E_{n \kappa}+\Sigma(r)\right] F_{n \kappa}(r),
\end{aligned}
$$

where $\Delta(r)=V(r)-S(r)$ and $\Sigma(r)=V(r)+S(r)$ are the difference and sum potentials, respectively.

In the presence of the spin symmetry ( i.e., $\Delta(r)=C_{s}=$ constant), one can eliminate $G_{n \kappa}(r)$ in Eq. (7a), with the aid of Eq. (7b), to obtain a second-order differential equation for the upper-spinor component:

$$
\left[-\frac{d^{2}}{d r^{2}}+\frac{\kappa(\kappa+1)}{r^{2}}+\frac{1}{\hbar^{2} c^{2}}\left(E_{n \kappa}+M c^{2}-C_{s}\right) \Sigma(r)\right] F_{n \kappa}(r)
$$




$$
=\frac{1}{\hbar^{2} c^{2}}\left[E_{n \kappa}^{2}-M^{2} c^{4}-C_{s}\left(E_{n \kappa}-M c^{2}\right)\right] F_{n \kappa}(r),
$$

and the lower-spinor component is obtained from Eq. (7a):

$$
G_{n \kappa}(r)=\frac{1}{M c^{2}+E_{n \kappa}-C_{s}}\left(\frac{d}{d r}+\frac{\kappa}{r}\right) F_{n \kappa}(r)
$$

where $E_{n \kappa} \neq-M c^{2}$, only real positive energy spectrum exist when $C_{s}=0$ (exact spin symmetry). On the other hand, in the presence of the pseudospin symmetry ( i.e., $\Sigma(r)=$ $C_{p s}=$ constant), one can eliminate $F_{n \kappa}(r)$ in Eq. $(7 \mathrm{~b})$, with the aid of Eq. (7a), to obtain a second-order differential equation for the lower-spinor component:

$$
\begin{gathered}
{\left[-\frac{d^{2}}{d r^{2}}+\frac{\kappa(\kappa-1)}{r^{2}}-\frac{1}{\hbar^{2} c^{2}}\left(M c^{2}-E_{n \kappa}+C_{p s}\right) \Delta(r] G_{n \kappa}(r)\right.} \\
=\frac{1}{\hbar^{2} c^{2}}\left[E_{n \kappa}^{2}-M^{2} c^{4}-C_{p s}\left(E_{n \kappa}+M c^{2}\right)\right] G_{n \kappa}(r),
\end{gathered}
$$

and the upper-spinor component $F_{n \kappa}(r)$ can be obtained from Eq. (7b) as

$$
F_{n \kappa}(r)=\frac{1}{M c^{2}-E_{n \kappa}+C_{p s}}\left(\frac{d}{d r}-\frac{\kappa}{r}\right) G_{n \kappa}(r),
$$

where $E_{n \kappa} \neq M c^{2}$, only real negative energy spectrum exist when $C_{p s}=0$ (exact pseudospin symmetry). The physical solution demands that the upper and lower radial components should satisfy the boundary conditions: $F_{n \kappa}(0)=G_{n \kappa}(0)=0$ and $F_{n \kappa}(\infty)=G_{n \kappa}(\infty)=0$.

\section{A. Spin symmetry Dirac-Yukawa problem}

At first, we investigate the spin symmetry in the form of $S U(2)$ by taking the $\Sigma(r)=$ $2 V(r) \rightarrow V_{Y}(r)$ [44] which can be easily reduced into the non-relativistic limit under a certain appropriate transformations. Equation (8) shows that the energy eigenvalues, $E_{n \kappa}$ is mainly dependent on the quantum numbers $n$ and $l$. For example, when $l \neq 0$, the states

with $j=l \pm 1 / 2$ are degenerate. The sum potential $\Sigma(r)$ in Eq. (8) is simply taken as the Yukawa potential,

$$
\Sigma(r)=-\frac{\Sigma_{0}}{r} e^{-\alpha r}, \Sigma_{0}=A>0
$$

which provides a simple Schrödinger-like equation in the form:

$$
\left[\frac{d^{2}}{d r^{2}}-\frac{\kappa(\kappa+1)}{r^{2}}-4 \alpha^{2} \nu_{1}^{2}+2 \alpha \omega_{1} \frac{A}{r} e^{-\alpha r}\right] F_{n \kappa}(r)=0, \kappa(\kappa+1)=l(l+1)
$$


where

$$
\nu_{1}^{2}=\frac{1}{4 \alpha^{2} \hbar^{2} c^{2}}\left(M c^{2}-E_{n \kappa}\right)\left(M c^{2}+E_{n \kappa}-C_{s}\right), \quad \omega_{1}=\frac{1}{2 \alpha \hbar^{2} c^{2}}\left[E_{n \kappa}+M c^{2}-C_{s}\right] A,
$$

with $\kappa$ values are given in Eq. (5). The exact analytic solution of Eq. (13) is difficult to find due to the centrifugal kinetic energy term $\kappa(\kappa+1) r^{-2}$ and the singularity of $r^{-1}$-type. Nonetheless, if $\kappa$ is not too large, the case of the vibrations of small amplitude about the minimum, we attempt to use the Greene-Aldrich [42] conventional approximation to deal with centrifugal term,

$$
\frac{1}{r^{2}} \approx 4 \alpha^{2} \frac{e^{-2 \alpha r}}{\left(1-e^{-2 \alpha r}\right)^{2}} .
$$

Introducing the new parameter, $x(r)=e^{-2 \alpha r} \in[0,1]$ and further substituting Eq. (15) into Eq. (13), we obtain

$$
\begin{gathered}
\left\{\frac{d^{2}}{d x^{2}}+\frac{(1-x)}{x(1-x)} \frac{d}{d x}\right. \\
\left.+\frac{\left[-\left(\nu_{1}^{2}+\omega_{1}\right) x^{2}+\left(2 \nu_{1}^{2}+\omega_{1}-\kappa(\kappa+1)\right) x-\nu_{1}^{2}\right]}{x^{2}(1-x)^{2}}\right\} F_{n \kappa}(x)=0,
\end{gathered}
$$

where we have inserted $F_{n \kappa}(r)=F_{n \kappa}(x)$. In order to clarify the parametric generalization of the NU method $[35,37,43]$, let us take the following general form of a Schrödinger-like equation written for any potential,

$$
\left[\frac{d^{2}}{d x^{2}}+\frac{\widetilde{\tau}(x)}{\sigma(x)} \frac{d}{d x}+\frac{\widetilde{\sigma}(x)}{\sigma^{2}(x)}\right] \psi_{n}(x)=0,
$$

satisfying the wave functions

$$
\psi_{n}(x)=\phi(x) y_{n}(x)
$$

In addition, the two polynomials

$$
\widetilde{\tau}(x)=c_{1}-c_{2} x
$$

and

$$
\sigma(x)=x\left(1-c_{3} x\right) \text { and } \tilde{\sigma}(x)=-\xi_{2} x^{2}+\xi_{1} x-\xi_{0},
$$

are at most of first- and second-degree, respectively. Comparing Eq. (17) with its counterpart Eq. (16), we obtain values for the constants $c_{i}(i=1,2,3)$ along with $\xi_{j}(j=0,1,2)$. Now, following the NU method [39] and making the substitution of Eqs. (19) and (20) leads to more general forms for the polynomials $\pi(z)$ and $\tau(z)$, the root $k$, the eigenvalues equation and the wave functions $\phi(z)$ and $y_{n}(z)$ all expressed in terms of the constants $c_{i}(i=4$, $5, \cdots, 13)$ as given in Appendix A. Hence, the task of computing the energy eigenvalues 
and the corresponding wave functions of Eq. (13) within the framework of the parametric generalization of the NU method becomes relatively easy and straightforward. It may be explained shortly in the following steps:

Firstly, we need to find the specific values for the parametric constants $c_{i}(i=4,5, \cdots, 13)$ by means of the relation A1 of Appendix A. The values of all these constants $c_{i}(i=1$, $2, \cdots, 13)$ together with $\xi_{j}(j=0,1,2)$ are therefore listed in Table 1 for the screened Coulomb potential model.

Secondly, by using the relations (A2-A5), the analytic forms of the essential polynomials $\pi(x)$ and $\tau(x)$ along with the root $k$, required by the NU method, can also be found as

$$
\begin{gathered}
\pi(x)=-\frac{x}{2}-\frac{1}{2}\left[\left(2 \kappa+1+2 \nu_{1}\right) x-2 \nu_{1}\right], \\
k=-\kappa(\kappa+1)+\omega_{1}-(2 \kappa+1) \nu_{1},
\end{gathered}
$$

and

$$
\tau(x)=1+2 \nu_{1}-2\left(1+\nu_{1}+\frac{1}{2}(2 \kappa+1)\right) x,
$$

where $\tau^{\prime}(x)<0$ must be satisfied in order to obtain physical solution according to the NU method [39].

Thirdly, we need to calculate the energy eigenvalues by means of the eigenvalue equation, relation A6 which gives

$$
(n+\kappa+1)^{2}+2(n+\kappa+1) \nu_{1}=\omega_{1} .
$$

Finally, after making use of Eq. (14), the above equation for the Yukawa potential can be expressed implicitly in terms of the energy $E_{n \kappa}$ as

$$
\begin{gathered}
\sqrt{\left(M c^{2}-E_{n \kappa}\right)\left(M c^{2}+E_{n \kappa}-C_{s}\right)}+\alpha \hbar c(n+\kappa+1)=\frac{\left(M c^{2}+E_{n \kappa}-C_{s}\right) A Z e^{2}}{2 \hbar c(n+\kappa+1)}, \\
M c^{2}>E_{n \kappa} \text { and } M c^{2}+E_{n \kappa}>C_{s}, n=0,1,2, \cdots
\end{gathered}
$$

where $\kappa \neq-(n+1)$ and $A=Z e^{2}$. The above energy equation can be also rearranged in a quadratic form (in relativistic units $\hbar=c=1$ ) as

$$
\left[1+\left(\frac{A}{N_{1}}\right)^{2}\right] E_{n \kappa}^{2}-2\left[S+\left(\frac{A}{N_{1}}\right)^{2} W\right] E_{n \kappa}+\left(\frac{A W}{N_{1}}\right)^{2}+\left(\frac{\alpha N_{1}}{2}\right)^{2}+(M+\alpha A) W=0
$$


where

$$
N_{1}=2(n+\kappa+1), W=C_{s}-M, S=\frac{1}{2}\left(C_{s}+\alpha A\right) .
$$

The two energy spectrum formula of the quadratic equation (26) is

$$
E_{n \kappa}^{ \pm}=\frac{\left(A^{2} W+S N_{1}^{2}\right) \pm \sqrt{\left(A^{2} W+S N_{1}^{2}\right)^{2}-\left(A^{2}+N_{1}^{2}\right)\left[\left(A W+\frac{1}{2} \alpha N_{1}^{2}\right)^{2}+M W N_{1}^{2}\right]}}{A^{2}+N_{1}^{2}}
$$

where $\left(A^{2} W+S N_{1}^{2}\right) \geq \sqrt{\left(A^{2}+N_{1}^{2}\right)\left[\left(A W+\frac{1}{2} \alpha N_{1}^{2}\right)^{2}+M W N_{1}^{2}\right]}$ for distinct particle and anti-particle real energy bound states $E_{n \kappa}^{p}=E_{n \kappa}^{+}$and $E_{n \kappa}^{a}=E_{n \kappa}^{-}$, respectively. Otherwise, in the case when the above inequality does not hold, we will have no bound state solutions (scattering states).

Figure 1 shows the ground state valence energy level of particle and antiparticle as a function of different values of the coupling constant $A$ and the screening parameter (range) of the potential $\alpha=0.01,0.02,0.05$ and 0.10 for two degenerate partners $(n=0, \kappa=1)$ and $(n=3, \kappa=-2)$ labelled as $\left(0 p_{1 / 2}, 3 p_{3 / 2}\right)$ of particle and antiparticle with the parameters choices of mass $M=5.0 \mathrm{fm}^{-1}$ and spin constant $C_{s}=4.9 \mathrm{fm}^{-1}$, as it should be expected, for a given value of $\alpha$ the bound state becomes sharply (slowly) deeper (more attractive) for particle (antiparticle) on increasing the coupling constant $A$ (heavy nucleus). On the decreasing the value of the range $\alpha$ (lower screening range), the energy goes to a more negative value for antiparticle and to a less positive value for particle (energy becomes more attractive). On the other hand, for a fixed value of $A$, the bound state energy becomes shallower on increasing $\alpha$ for particle. It is seen that in the limit of a very short-ranged potential $(\alpha \rightarrow 0)$, the potential approaches the $\delta$-function limit that can bind particles and antiparticle stronger than finite-ranged potentials (1). Furthermore, Fig. 2 shows the variation of ground state valence energy level of particle and antiparticle with the spin constant $C_{s}$ for several values of the spin-orbit $\kappa=1,3$ and 5 with special choices of parameters $M=5.0$ $\mathrm{fm}^{-1}, \alpha=0.1 \mathrm{fm}^{-1}$ and $A=1$. A very careful inspection for both numerical results and Fig. 2a shows that there is a small energy difference between the states $\kappa=1,3$ and 5 although the values of spin constant $C_{s}$ increases in the range $11.4 \mathrm{fm}^{-1} \leq C_{s} \leq 20 \mathrm{fm}^{-1}$, i.e., the energy spectrum is not sensitive to the influence of $\kappa$ in the aforementioned range. With the increasing $\kappa$ value, we see that $E_{0 \kappa}^{+}$fan out toward the stronger positive energy spectrum for the given range $-20 \mathrm{fm}^{-1} \leq C_{s} \leq 8.8 \mathrm{fm}^{-1}$. The scattering (not bound) states 
could be seen in the range $8.8 \mathrm{fm}^{-1} \leq C_{s} \leq 11.3 \mathrm{fm}^{-1}$ for $\kappa=1,3$ and 5 . The physical explanation to Figure 2a is being illustrated as follows. It is seen in Table 2 that the energy spectrum (positive and negative) of Eq. (25) is entirely dependent on the choice of $C_{s}$, i.e., $E_{0 \kappa}^{+}=E_{0 \kappa}^{+}\left(C_{s}\right)$. For example, in the single electron interaction with nucleus $Z e$ (units $\hbar=c=e=1$ ), we may have two essential requirements: (a) $M \geq E_{n \kappa}$ and $M+E_{n \kappa} \geq C_{s}$, in which both impose a restriction on the choice of the range values of the permissible spin symmetry constant $C_{s}$ are in the interval $-20 \mathrm{fm}^{-1} \leq C_{s} \leq 8.8 \mathrm{fm}^{-1}$ with a requirement that energy spectrum be real along with $\left|E_{n \kappa}\right|<M$ and (b) $M \leq E_{n \kappa}$ and $M+E_{n \kappa} \leq C_{s}$ which is possible for $11.4 \mathrm{fm}^{-1} \leq C_{s} \leq 20 \mathrm{fm}^{-1}$ where $E_{n \kappa}>M$. In the two cases we have taken the spin-orbit quantum number $\kappa=1,3$ and 5 . While taking $\kappa=1,3,5,7$ and 9, $C_{s}$ lies in the two intervals: $-20 \mathrm{fm}^{-1} \leq C_{s} \leq 8.0 \mathrm{fm}^{-1}$ and $12.2 \mathrm{fm}^{-1} \leq C_{s} \leq 20$ $\mathrm{fm}^{-1}$. Nonetheless, it is necessary to choose the physical solution as shown in case (a) since it is consistent with the exact spin symmetry when $C_{s}=0$ (i.e., $\left.S(r)=V(r)\right)\left(\left|E_{n \kappa}\right|<M\right)$ in which the energy levels become very sensitive to the influence of $\kappa$ as usually expected. Table 2 supports our choice of the allowed range $-20 \mathrm{fm}^{-1} \leq C_{s} \leq 8.8 \mathrm{fm}^{-1}$. Moreover, we find out that the second case is not sensitive to the influence of $\kappa$. Thus, for our choice of $C_{s}=4.9 \mathrm{fm}^{-1}$ which is falling in (a), we present all ground energy spectrum including four states: $E_{0,1}=4.78641 \mathrm{fm}^{-1}, E_{0,2}=4.94209 \mathrm{fm}^{-1}, E_{0,3}=4.98904 \mathrm{fm}^{-1}$ and $E_{0,4}=4.99998$ $f m^{-1}$.

The results presented in Fig. $2 \mathrm{~b}$ and Table 2 show that the energy difference of antiparticle between the states $\kappa=1,3$ and 5 shows a slight change although the values of spin constant $C_{s}$ increases in the interval $10.6 \mathrm{fm}^{-1} \leq C_{s} \leq 20 \mathrm{fm}^{-1}$ (same as in Fig. 2a). This range is forbidden since $E_{0, \kappa}^{-}<-M$. As $\kappa$ decreasing, $E_{0 \kappa}^{-}$fan out toward the stronger (deeper) negative energy spectrum when $-16 \mathrm{fm}^{-1} \leq C_{s} \leq-0.1 \mathrm{fm}^{-1}$ ( $E_{0, \kappa}^{-}$is very strongly negative, forbidden). In the intervals $0 \mathrm{fm}^{-1} \leq C_{s} \leq 4.9 \mathrm{fm}^{-1}, E_{0, \kappa}^{-}<M$ (negative, permissible), $5.0 \mathrm{fm}^{-1} \leq C_{s} \leq 9.6 \mathrm{fm}^{-1}, E_{0, \kappa}^{-}<M$ (positive, permissible) while 9.7 $\mathrm{fm}^{-1} \leq C_{s} \leq 10.6 \mathrm{fm}^{-1}$ (energy is complex). The variation of the ground negative energy level with $C_{s}$ contains forbidden values of $C_{s}$ that gives $E_{0, \kappa} \neq-M$ which results in an infinity wave function. The range values of the allowed $C_{s}$ are in the interval $0 \mathrm{fm}^{-1}<$ $C_{s} \leq 9.6 \mathrm{fm}^{-1}$ with $E_{0, \kappa} \neq-M$ when $C_{s}=0$.

Next, in order to establish the wave functions $F_{n \kappa}(r)$ of Eq. (8), the relations (A7-A10) 
are used. Firstly, we calculate the first part of the wave functions,

$$
\phi(x)=x^{\nu_{1}}(1-x)^{\kappa+1}, \nu_{1}>0
$$

The weight function takes the form

$$
\rho(x)=x^{2 \nu_{1}}(1-x)^{2 \kappa+1} .
$$

which can generate the second part of the wave functions,

$$
y_{n}(x) \sim x^{-2 \nu_{1}}(1-x)^{-(2 \kappa+1)} \frac{d^{n}}{d x^{n}}\left[x^{n+2 \nu_{1}}(1-x)^{n+2 \kappa+1}\right] \approx P_{n}^{\left(2 \nu_{1}, 2 \kappa+1\right)}(1-2 x),
$$

where $P_{n}^{(a, b)}(1-2 x)$ is the orthogonal Jacobi polynomials [45,46]. Finally, the upper spinor component $F_{n \kappa}(x)$ for any arbitrary $\kappa$ can be obtained by means of Eq. (18) as

$$
\begin{gathered}
F_{n \kappa}(r)=\mathcal{N}_{n \kappa} e^{-2 \nu_{1} \alpha r}\left(1-e^{-2 \alpha r}\right)^{\kappa+1} P_{n}^{\left(2 \nu_{1}, 2 \kappa+1\right)}\left(1-2 e^{-2 \alpha r}\right), \\
P_{n}^{\left(2 \nu_{1}, 2 \kappa+1\right)}\left(1-2 e^{-2 \alpha r}\right)=\frac{\Gamma\left(n+2 \nu_{1}+1\right)}{\Gamma\left(2 \nu_{1}+1\right) n !}{ }_{2} F_{1}\left(-n, n+2\left(\nu_{1}+\kappa+1\right) ; 1+2 \nu_{1} ; e^{-2 \alpha r}\right),
\end{gathered}
$$

where the normalization constants $\mathcal{N}_{n \kappa}$ are calculated in Appendix B.

The derivative relation of the hypergeometric function,

$$
\frac{d}{d x}\left[{ }_{2} F_{1}(a ; b ; c ; x)\right]=\left(\frac{a b}{c}\right){ }_{2} F_{1}(a+1 ; b+1 ; c+1 ; x),
$$

is usually used to calculate the corresponding lower-component $G_{n \kappa}(r)$ by means of Eq. (9):

$$
\begin{gathered}
G_{n \kappa}(r)=\frac{\mathcal{N}_{n \kappa}}{M c^{2}+E_{n \kappa}-C_{s}}\left(\frac{2 \alpha(\kappa+1) e^{-2 \alpha r}}{1-e^{-2 \alpha r}}-2 \alpha \nu_{1}+\frac{\kappa}{r}\right) F_{n \kappa}(r) \\
+\mathcal{N}_{n \kappa} \frac{2 n \alpha\left(n+2 \nu_{1}+2 \kappa+2\right)}{\left(M c^{2}+E_{n \kappa}-C_{s}\right)\left(1+2 \nu_{1}\right)}\left(1-e^{-2 \alpha r}\right)^{\kappa+1}\left(e^{-2 \alpha r}\right)^{\nu_{1}+1} \\
\quad \times{ }_{2} F_{1}\left(-n+1 ; n+2\left(\nu_{1}+\kappa+1\right)+1 ; 2\left(1+\nu_{1}\right) ; e^{-2 \alpha r}\right) .
\end{gathered}
$$

The hypergeometric series ${ }_{2} F_{1}\left(-n+1 ; n+2\left(\nu_{1}+\kappa+1\right)+1 ; 2\left(1+\nu_{1}\right) ; e^{-\alpha r}\right)$ is terminated for $n=0$ and thus does not diverge for all values of real parameters $\nu_{1}$ and $\kappa+1$. In relativistic units $\hbar=c=1, E_{n \kappa} \neq-M$, i.e., negative energy states are forbidden, when $C_{s}=0$ in which the positive energy solution of Eq. (25) is required. Therefore, the negative solution is not desirable, see Fig. 2b..

In Fig. 3, we plot the upper and lower spinor wave functions of ground $0 p_{1 / 2}$ and first excited $1 p_{1 / 2}$ states for (a) particle and (b) antiparticle with $\kappa=1$. The choices of parameters 
$M=5.0 \mathrm{fm}^{-1}, \mathrm{C}_{s}=4.9 \mathrm{fm}^{-1}, \alpha=0.1 \mathrm{fm}^{-1}$ and $A=1$ are used. In case of the particle (positive energy), the upper and lower spinor wave functions of the ground (first excited) state are found to be similar in shape. It is noted that the amplitude of the upper wave function $F_{01}^{+}(r)\left(F_{11}^{+}(r)\right)$ is nearly three times larger than the lower wave function $G_{01}^{+}(r)$ $\left(G_{11}^{+}(r)\right)$. Further, the amplitude of the ground state wave function $F_{01}^{+}(r)$ is nearly two times larger than the first excited state wave function $F_{11}^{+}(r)$. The range of the upper component is wider than the lower component of the wave function and the range of the first excited state is wider than the range of the ground state wave function.

Let us now study the nonrelativistic case. Making the appropriate changes: $\nu_{1}^{2} \rightarrow \varepsilon_{n l}^{2}=$ $-\left(2 m E_{n l} / 4 \alpha^{2} \hbar^{2}\right), \omega_{1} \rightarrow \gamma_{1}=\left(m A / \alpha \hbar^{2}\right)$ and $\kappa(\kappa+1) \rightarrow l(l+1)$ in Eqs. (16), (21)-(24) and (29)-(32) together with Table 1, we can easily obtain the energy spectrum of the Schrödinger equation for the Yukawa potential model:

$$
\varepsilon_{n l}=\frac{\gamma_{1}}{2(n+l+1)}-\frac{(n+l+1)}{2}
$$

which can be explicitly expressed as

$$
E_{n l}=-\frac{\hbar^{2}}{2 m}\left[\frac{m A}{\hbar^{2}(n+l+1)}-(n+l+1) \alpha\right]^{2},
$$

and the radial wave functions:

$$
\begin{gathered}
R_{n l}(r)=r^{-1} u_{n l}(r)=\mathcal{N}_{n l} r^{-1} e^{-\epsilon_{n l} r}\left(1-e^{-2 \alpha r}\right)^{l+1} P_{n}^{\left(2 \varepsilon_{n l}, 2 l+1\right)}\left(1-2 e^{-2 \alpha r}\right) \\
=\mathcal{N}_{n l} \frac{\Gamma\left(n+2 \varepsilon_{n l}+1\right)}{\Gamma\left(2 \varepsilon_{n l}+1\right) n !} e^{-\epsilon_{n l} r}\left(1-e^{-2 \alpha r}\right)^{l+1}{ }_{2} F_{1}\left(-n, n+2\left(\varepsilon_{n l}+l+1\right) ; 1+2 \varepsilon_{n l} ; e^{-2 \alpha r}\right),
\end{gathered}
$$

where $\epsilon_{n l}=2 \alpha \varepsilon_{n l}=\left[\frac{m A}{\hbar^{2}(n+l+1)}-(n+l+1) \alpha\right]$ and the normalization constants $\mathcal{N}_{n l}$ are carried out in Appendix B.

\section{B. Pseudospin symmetry Dirac-Yukawa problem}

From Eq. (10), we can see that the energy eigenvalues depend mainly only on $n$ and $\tilde{l}$, i.e., $E_{n \kappa}=E(n, \tilde{l}(\widetilde{l}+1))$. For $\widetilde{l} \neq 0$, the states with $j=\widetilde{l} \pm 1 / 2$ are degenerate. This is a $S U(2)$ pseudospin symmetry. We impose $\Delta(r)$ as the Yukawa potential model given in (1):

$$
\Delta(r)=-\frac{\Delta_{0}}{r} e^{-\alpha r}, \Delta_{0}=A>0
$$


leading to a Schrödinger-like equation in the form:

$$
\left[\frac{d^{2}}{d r^{2}}-\frac{\kappa(\kappa-1)}{r^{2}}-4 \alpha^{2} \nu_{2}^{2}+2 \alpha \omega_{2} \frac{A}{r} e^{-\alpha r}\right] G_{n \kappa}(r)=0
$$

with

$$
\nu_{2}^{2}=\frac{1}{4 \alpha^{2} \hbar^{2} c^{2}}\left(M c^{2}+E_{n \kappa}\right)\left(M c^{2}-E_{n \kappa}+C_{p s}\right), \quad \omega_{2}=\frac{1}{2 \alpha \hbar^{2} c^{2}}\left[E_{n \kappa}-M c^{2}-C_{p s}\right] A,
$$

where $\kappa(\kappa-1)=\widetilde{l}(\widetilde{l}+1)$ satisfying Eq. (6). We follow the same procedures of solutions discussed before to obtain a Dirac equation satisfying $G_{n \kappa}(r)$,

$$
\begin{gathered}
\left\{\frac{d^{2}}{d x^{2}}+\frac{(1-x)}{x(1-x)} \frac{d}{d x}\right. \\
\left.+\frac{\left[-\left(\nu_{2}^{2}+\omega_{2}\right) x^{2}+\left(2 \nu_{2}^{2}+\omega_{2}-\kappa(\kappa-1)\right) x-\nu_{2}^{2}\right]}{x^{2}(1-x)^{2}}\right\} G_{n \kappa}(x)=0 .
\end{gathered}
$$

To avoid repetition in the solution of Eq. (40), a careful inspection for the relationship between the present set of parameters $\left(\omega_{2}, \nu_{2}^{2}\right)$ and the previous one $\left(\omega_{1}, \nu_{1}^{2}\right)$ tells us that the negative energy solution for pseudospin symmetry, where $S(r) \sim-V(r)$, can be obtained directly from the spin symmetric solution by using the following parameter mapping [40,47]:

$F_{n \kappa}(r) \leftrightarrow G_{n \kappa}(r), \kappa \rightarrow \kappa-1, V(r) \rightarrow-V(r)$ (i.e., $A \rightarrow-A$ ), $E_{n \kappa} \rightarrow-E_{n \kappa}$ and $C_{s} \rightarrow-C_{p s}$.

Further, the constants in the case of pseudospin symmetry concept are listed in Table 1. Applying the above transformations to Eqs. (21)-(24) leading to the following pseudospin symmetric energy equation,

$$
(n+\kappa)^{2}+2(n+\kappa) \nu_{2}=\omega_{2}
$$

Finally, with the aid of Eq. (39), Eq. (42) can be also expressed in terms of the energy,

$$
\begin{gathered}
\sqrt{\left(M c^{2}+E_{n \kappa}\right)\left(M c^{2}-E_{n \kappa}+C_{p s}\right)}+\alpha \hbar c(n+\kappa)=\frac{\left(E_{n \kappa}-M c^{2}-C_{p s}\right) A}{2 \hbar c(n+\kappa)}, \\
M c^{2}>-E_{n \kappa} \text { and } M c^{2}+C_{p s}>E_{n \kappa}, n=0,1,2, \cdots
\end{gathered}
$$

where $\kappa \neq-n$. When $C_{p s}=0$ (exact pseudospin symmetry, $S(r)=-V(r)$ case) then we require $-M<E_{n \kappa}<M$. The above pseudospin energy equation can be rearranged in a quadratic form $(\hbar=c=1)$ :

$$
\left[1+\left(\frac{A}{N_{2}}\right)^{2}\right] E_{n \kappa}^{2}-2\left[T+\left(\frac{A}{N_{2}}\right)^{2} U\right] E_{n \kappa}+\left(\frac{A U}{N_{2}}\right)^{2}+\left(\frac{\alpha N_{2}}{2}\right)^{2}-(M-\alpha A) U=0
$$


where

$$
N_{2}=2(n+\kappa), U=C_{p s}+M, T=\frac{1}{2}\left(C_{p s}+\alpha A\right)
$$

The above quadratic energy equation can be easily obtained by means of Eq. (25) through making the replacements: $n+\kappa+1 \rightarrow n+\kappa, A \rightarrow-A, E_{n \kappa} \rightarrow-E_{n \kappa}$ and $C_{s} \rightarrow-C_{p s}$. The two energy solutions of the quadratic equation (44) can be obtained as

$$
E_{n \kappa}^{ \pm}=\frac{\left(A^{2} U+T N_{2}^{2}\right) \pm \sqrt{\left(A^{2} U+T N_{2}^{2}\right)^{2}-\left(A^{2}+N_{2}^{2}\right)\left[\left(A U+\frac{1}{2} \alpha N_{2}^{2}\right)^{2}-M U N_{2}^{2}\right]}}{A^{2}+N_{2}^{2}}
$$

where $\left(A^{2} U+T N_{2}^{2}\right)>\sqrt{\left(A^{2}+N_{2}^{2}\right)\left[\left(A U+\frac{1}{2} \alpha N_{2}^{2}\right)^{2}-M U N_{2}^{2}\right]}$ for distinct particle $E_{n \kappa}^{p}$ and anti-particle $E_{n \kappa}^{a}$ real bound state energies.

On the basis of pseudospin symmetry, Fig. 4 shows the ground state hole energy level of particle and antiparticle for different values of the coupling constant $A$ and the screening parameter (range) of the potential $\alpha=0.01,0.02,0.05$ and 0.10 for two degenerate partners $(n=0, \kappa=2)$ and $(n=1, \kappa=-3)$ labelled as $\left(0 d_{3 / 2}, 1 d_{5 / 2}\right)$ with $M=5.0 \mathrm{fm}^{-1}$ and $C_{p s}=-5.0 \mathrm{fm}^{-1}$. In this exploratory investigation, as it should be expected, for a given value of $\alpha$ the bound state becomes slowly (sharply) deeper for particle (antiparticle), i.e., it becomes more (less) attractive, on increasing the coupling constant $A$ (heavy nucleus). On increasing the value of the screening parameter $\alpha$, the particle (antiparticle) becomes more (less) attractive as $A$ increasing. Therefore, in the pseudospin symmetry case, the antiparticle is less attractive to heavier nuclei while in the spin symmetry case the particle is more attractive to heavier nuclei. In addition, increasing the value of screening parameter $\alpha$ in both particle and antiparticle leads to less attractive interaction with heavier nucleus.

Furthermore, Fig. 5 shows the variation of the ground state hole energy for particle and antiparticle as a function of different values of pseudospin constant $C_{p s}$ plotted for several values of the spin-orbit $\kappa=1,3$ and 5 . We take the set of parameters $M=5.0 \mathrm{fm}^{-1}, \alpha=0.1$ $\mathrm{fm}^{-1}$ and $A=1$. The results presented in Fig. 5a show that the energy difference of particle between the states $\kappa=1,3$ and 5 is almost same although the values of spin constant $C_{p s}$ increases in the interval $-8 \mathrm{fm}^{-1} \leq C_{p s} \leq 20 \mathrm{fm}^{-1}$. With an increasing of $\kappa$ value, $E_{0 \kappa}^{+}$ is seen to fan out toward the stronger negative energy spectrum (more attractive) when $-20 \mathrm{fm}^{-1} \leq C_{p s} \leq-13 \mathrm{fm}^{-1}$. The scattering states could be seen in the short interval $-12 \mathrm{fm}^{-1} \leq C_{p s} \leq-8 \mathrm{fm}^{-1}$. Figure $5 \mathrm{~b}$ shows that the energy difference of antiparticle between the states $\kappa=1,3$ and 5 is almost same although the values of spin constant $C_{p s}$ 
increases in the interval $-20 \mathrm{fm}^{-1} \leq C_{p s} \leq-11 \mathrm{fm}^{-1}$ (i.e., antiparticle energy state is not sensitive to spin-orbit quantum number $\kappa$ ). With the $\kappa$ increasing, $E_{0 \kappa}^{-}$fan out toward the stronger (deeper) negative energy spectrum when $-8 \mathrm{fm}^{-1} \leq C_{p s} \leq 20 \mathrm{fm}^{-1}$. The scattering states could be seen in the short interval $-11 \mathrm{fm}^{-1} \leq C_{p s} \leq-8 \mathrm{fm}^{-1}$. In the presence of pseudospin, we consider the physical case where the energy has to be negative (i.e., $E_{n \kappa} \neq M$ when $C_{p s}=0$ ) which is simply the case of antiparticle. As seen in Fig. 5b, the range of the allowed pseudospin constant $C_{p s}$ falls in the range $-8 \mathrm{fm}^{-1}<C_{p s}<20$ $f m^{-1}$ in which $E_{n \kappa}^{-}$is sensitive to the influence of $\kappa$ and also energy results satisfy the condition $\left|E_{n \kappa}\right|<M$. Consequently, we choose $C_{p s}=-5.0 \mathrm{fm}^{-1}$ in the present numerical calculations.

We present the essential procedures in calculating the wave functions. The first part of the wave functions is

$$
\phi(x)=x^{\nu_{2}}(1-x)^{\kappa}, \nu_{1}>0
$$

and the weight function is

$$
\rho(x)=x^{2 \nu_{2}}(1-x)^{2 \kappa-1}
$$

and this generates the second part of the wave functions,

$$
y_{n}(x) \sim x^{-2 \nu_{2}}(1-x)^{-(2 \kappa-1)} \frac{d^{n}}{d x^{n}}\left[x^{n+2 \nu_{2}}(1-x)^{n+2 \kappa-1}\right] \approx P_{n}^{\left(2 \nu_{2}, 2 \kappa-1\right)}(1-2 x)
$$

Finally, the lower spinor component $G_{n \kappa}(x)$ for arbitrary $\kappa$ can be obtained by means of Eq. (18) as

$$
\begin{gathered}
G_{n \kappa}(r)=\mathcal{N}_{n \kappa} e^{-2 \nu_{2} \alpha r}\left(1-e^{-2 \alpha r}\right)^{\kappa} P_{n}^{\left(2 \nu_{2}, 2 \kappa-1\right)}\left(1-2 e^{-2 \alpha r}\right), \\
P_{n}^{\left(2 \nu_{2}, 2 \kappa-1\right)}\left(1-2 e^{-2 \alpha r}\right)=\frac{\Gamma\left(n+2 \nu_{2}+1\right)}{\Gamma\left(2 \nu_{2}+1\right) n !}{ }_{2} F_{1}\left(-n, n+2\left(\nu_{2}+\kappa\right) ; 1+2 \nu_{2} ; e^{-2 \alpha r}\right) .
\end{gathered}
$$

The upper-component $F_{n \kappa}(r)$ can be calculated from Eq. (11) as follows

$$
\begin{gathered}
F_{n \kappa}(r)=\frac{\mathcal{N}_{n \kappa}}{\left(M c^{2}-E_{n \kappa}+C_{p s}\right)}\left(\frac{2 \alpha \kappa e^{-2 \alpha r}}{1-e^{-2 \alpha r}}-2 \alpha \nu_{2}-\frac{\kappa}{r}\right) G_{n \kappa}(r) \\
+\mathcal{N}_{n \kappa} \frac{2 n \alpha\left(n+2 \nu_{2}+2 \kappa\right)}{\left(M c^{2}-E_{n \kappa}+C_{p s}\right)\left(1+2 \nu_{2}\right)}\left(1-e^{-2 \alpha r}\right)^{\kappa}\left(e^{-2 \alpha r}\right)^{\nu_{2}+1} \\
\times{ }_{2} F_{1}\left(-n+1 ; n+2\left(\nu_{2}+\kappa\right)+1 ; 2\left(1+\nu_{2}\right) ; e^{-2 \alpha r}\right) .
\end{gathered}
$$

In relativistic units $\hbar=c=1, E_{n \kappa} \neq M$, i.e., positive energy states are forbidden, when $C_{p s}=0$ in which the negative (antiparticle) energy solution of Eq. (43) is required. 
Therefore, the positive solution is not desirable, see Fig. 5a. The hypergeometric series ${ }_{2} F_{1}\left(-n+1 ; n+2\left(\nu_{2}+\kappa\right)+1 ; 2\left(1+\nu_{2}\right) ; e^{-\alpha r}\right)$ terminates for $n=0$ and thus does not diverge for all values of real parameters $\nu_{2}$ and $\kappa$.

In Fig. 6, we plot the upper and lower spinor wave functions of ground $0 d_{3 / 2}$ and first excited $1 d_{3 / 2}$ states for (a) particle and (b) antiparticle with $\kappa=2$. The set of parameters $M=5.0 \mathrm{fm}^{-1}, \mathrm{C}_{p s}=-5.0 \mathrm{fm}^{-1}, \alpha=0.1 \mathrm{fm}^{-1}$ and $A=1$ are used.

\section{A FEW SPECIAL CASES}

Let us study four special cases. At first, we study the nonrelativistic (SchrödingerYukawa) case by setting $C_{s}=0$ (exact spin symmetry limit) and making the changes $\kappa \rightarrow l$, $M+E_{n \kappa} \rightarrow 2 \mu / \hbar^{2}$ and $M-E_{n \kappa} \rightarrow-E_{n \kappa}$. Hence, from Eq. (25), it follows that

$$
E_{n l}=-\frac{2 \mu}{\hbar^{2}}\left[\frac{A_{0}}{2(n+l+1)}-\frac{\hbar^{2}}{2 \mu}(n+l+1) \alpha\right]^{2}, n=0,1,2, \cdots, l=0,1,2, \cdots
$$

Further, in the limit when $\alpha=0$, the above result reduces to the well-known spectrum for the nonrelativistic Coulombic field, $E_{n l}^{(C)}=-\frac{\mu A_{0}^{2}}{2 \hbar^{2}(n+l+1)^{2}}$ with a wave functions $R_{n l}(r)=$ $r^{-1} \chi_{n l}(r)=r^{-1} e^{-\beta r} r^{l+1} L_{n}^{(2 l+1)}(2 \beta r)$ with $\beta=\frac{\mu A}{\hbar^{2}(n+l+1)}>0$, and $L_{n}^{s}(x)$ is the Laguarre function $[22]$.

Second, spin symmetry Dirac-Coulomb $(\alpha \rightarrow 0)$ case

$$
\sqrt{\left(M c^{2}-E_{n \kappa}\right)\left(M c^{2}+E_{n \kappa}-C_{s}\right)}=\frac{\left(M c^{2}+E_{n \kappa}-C_{s}\right) A}{2 \hbar c(n+\kappa+1)}, n=0,1,2, \cdots,
$$

The above energy equation can be rearranged in a quadratic form $(\hbar=c=1)$ :

$$
\left[1+\left(\frac{A}{N_{1}}\right)^{2}\right] E_{n \kappa}^{2}-\left[C_{s}+2\left(\frac{A}{N_{1}}\right)^{2} W\right] E_{n \kappa}+\left(\frac{A W}{N_{1}}\right)^{2}+M W=0,
$$

and the two energy solutions of the above equation can be obtained as

$$
E_{n \kappa}^{ \pm}=\frac{\left(2 A^{2} W+C_{s} N_{1}^{2}\right) \pm \sqrt{\left(2 A^{2} W+C_{s} N_{1}^{2}\right)^{2}-4 W\left(A^{2}+N_{1}^{2}\right)\left[A^{2} W+M N_{1}^{2}\right]}}{2\left(A^{2}+N_{1}^{2}\right)},
$$

In the limitation of pseudospin symmetry, the Dirac-Ykawa problem reduces to DiracCoulomb problem when $\alpha \rightarrow 0$,

$$
\sqrt{\left(M c^{2}+E_{n \kappa}\right)\left(M c^{2}-E_{n \kappa}+C_{p s}\right)}=\frac{\left(E_{n \kappa}-M c^{2}-C_{p s}\right) A}{2 \hbar c(n+\kappa)}, n=0,1,2, \cdots,
$$


and it can be rearranged in a quadratic form $(\hbar=c=1)$ as

$$
\left[1+\left(\frac{A}{N_{2}}\right)^{2}\right] E_{n \kappa}^{2}-\left[C_{p s} T+2\left(\frac{A}{N_{2}}\right)^{2} U\right] E_{n \kappa}+\left(\frac{A U}{N_{2}}\right)^{2}-M U=0 .
$$

Thus, the two energy solutions of the above equation can be readily obtained as

$$
E_{n \kappa}^{ \pm}=\frac{\left(2 A^{2} U+C_{p s} N_{2}^{2}\right) \pm \sqrt{\left(2 A^{2} U+C_{p s} N_{2}^{2}\right)^{2}-4 U\left(A^{2}+N_{2}^{2}\right)\left[A^{2} U-M N_{2}^{2}\right]}}{2\left(A^{2}+N_{2}^{2}\right)},
$$

Third, on the base of the exact spin symmetry $\left(C_{s}=0\right)$, the energy equation for DiracYukawa problem becomes (in units $\hbar=c=1$ )

$$
\sqrt{M-E_{n \kappa}}+\alpha(n+\kappa+1)=\frac{A \sqrt{M+E_{n \kappa}}}{2(n+\kappa+1)}, \quad\left|E_{n \kappa}\right|<M, n=0,1,2, \cdots,
$$

with two energy solutions of the quadratic equation (25) can be obtained as

$$
E_{n \kappa}^{ \pm}=\frac{\left(\alpha N_{1}^{2}-2 M A\right) A \pm \sqrt{\left(\alpha N_{1}^{2}-2 M A\right)^{2} A^{2}-\left(A^{2}+N_{1}^{2}\right)\left[\left(\alpha N_{1}^{2}-2 M A\right)^{2}-\left(2 M N_{1}\right)^{2}\right]}}{2\left(A^{2}+N_{1}^{2}\right)},
$$

where $N_{1}$ is defined in Eq. (27). From Eqs. (32) and (33), the upper and lower wave functions are

$$
F_{n \kappa}(r)=\mathcal{N}_{n \kappa} \frac{\Gamma(n+2 \gamma+1)}{\Gamma(2 \gamma+1) n !} e^{-2 \gamma \alpha r}\left(1-e^{-2 \alpha r}\right)^{\kappa+1}{ }_{2} F_{1}\left(-n, n+2(\gamma+\kappa+1) ; 1+2 \gamma ; e^{-2 \alpha r}\right),
$$

and

$$
\begin{gathered}
G_{n \kappa}(r)=\frac{\mathcal{N}_{n \kappa}}{M+E_{n \kappa}}\left(\frac{2 \alpha(\kappa+1) e^{-2 \alpha r}}{1-e^{-2 \alpha r}}-2 \alpha \gamma+\frac{\kappa}{r}\right) F_{n \kappa}(r) \\
+\mathcal{N}_{n \kappa} \frac{2 n \alpha(n+2 \gamma+2 \kappa+2)}{\left(M+E_{n \kappa}\right)(1+2 \gamma)}\left(1-e^{-2 \alpha r}\right)^{\kappa+1}\left(e^{-2 \alpha r}\right)^{\gamma+1} \\
\times{ }_{2} F_{1}\left(-n+1 ; n+2(\gamma+\kappa+1)+1 ; 2(1+\gamma) ; e^{-\alpha r}\right), M \neq-E_{n \kappa}
\end{gathered}
$$

respectively, where $\gamma=\frac{1}{2 \alpha} \sqrt{M^{2}-E_{n \kappa}^{2}}$.

In view of the exact pseudospin symmetry $\left(C_{p s}=0\right)$, the energy equation for DiracYukawa problem reads $(\hbar=c=1)$

$$
\sqrt{M^{2}-E_{n \kappa}^{2}}+\alpha(n+\kappa)=\frac{\left(E_{n \kappa}-M\right) A}{2(n+\kappa)}, n=0,1,2, \cdots,
$$

where $\left|E_{n \kappa}\right|<M$ and with two energy solutions:

$$
E_{n \kappa}^{ \pm}=\frac{\left(\alpha N_{2}^{2}+2 M A\right) A \pm \sqrt{\left(\alpha N_{2}^{2}+2 M A\right)^{2} A^{2}-\left(A^{2}+N_{2}^{2}\right)\left[\left(\alpha N_{2}^{2}+2 M A\right)^{2}-\left(2 M N_{2}\right)^{2}\right]}}{2\left(A^{2}+N_{2}^{2}\right)},
$$


The lower and upper-component wave functions are

$$
G_{n \kappa}(r)=\mathcal{N}_{n \kappa} \frac{\Gamma(n+2 \gamma+1)}{\Gamma(2 \gamma+1) n !} e^{-2 \gamma \alpha r}\left(1-e^{-2 \alpha r}\right)^{\kappa}{ }_{2} F_{1}\left(-n, n+2(\gamma+\kappa) ; 1+2 \gamma ; e^{-2 \alpha r}\right),
$$

and

$$
\begin{gathered}
F_{n \kappa}(r)=\frac{\mathcal{N}_{n \kappa}}{\left(M c^{2}-E_{n \kappa}\right)}\left(\frac{2 \alpha \kappa e^{-2 \alpha r}}{1-e^{-2 \alpha r}}-2 \alpha \gamma-\frac{\kappa}{r}\right) G_{n \kappa}(r) \\
+\mathcal{N}_{n \kappa} \frac{2 n \alpha(n+2 \gamma+2 \kappa)}{\left(M c^{2}-E_{n \kappa}\right)(1+2 \gamma)}\left(1-e^{-2 \alpha r}\right)^{\kappa}\left(e^{-2 \alpha r}\right)^{\gamma+1} \\
\quad \times{ }_{2} F_{1}\left(-n+1 ; n+2(\gamma+\kappa)+1 ; 2(1+\gamma) ; e^{-\alpha r}\right) .
\end{gathered}
$$

respectively.

Fourth, let us find the analytic solution of the Yukawa plus an added centrifugal-like term, i.e., $V(r)=-\frac{A}{r} e^{-\alpha r}+\frac{D}{r^{2}}$. The aim behind this choice is to remove the degeneracy of energy for various states. In view of spin symmetry, after inserting $\Sigma(r)=-\frac{A}{r} e^{-\alpha r}+\frac{D}{r^{2}}$ into Eq. (8), we obtain

$$
\left[\frac{d^{2}}{d r^{2}}-\frac{\kappa^{\prime}\left(\kappa^{\prime}+1\right)}{r^{2}}-4 \alpha^{2} \nu_{1}^{2}+2 \alpha \omega_{1} \frac{A}{r} e^{-\alpha r}\right] F_{n \kappa}(r)=0
$$

and, hence, the energy spectrum can be obtained from Eq. (28) by making the change $\kappa \rightarrow \kappa^{\prime}$ as

$$
\sqrt{\left(M-E_{n \kappa}\right)\left(M+E_{n \kappa}-C_{s}\right)}+\alpha\left(n+\kappa^{\prime}+1\right)=\frac{\left(M+E_{n \kappa}-C_{s}\right) A}{2\left(n+\kappa^{\prime}+1\right)}, \kappa^{\prime} \neq-(n+1)
$$

where

$$
\kappa^{\prime}=-\frac{1}{2}+\frac{1}{2} \sqrt{(2 \kappa+1)^{2}+4 D\left(M+E_{n \kappa^{\prime}}-C_{s}\right)} .
$$

Further, the upper and lower wave functions can be obtained simply via Eqs. (32) and (33) as

$$
F_{n \kappa}(r)=\mathcal{N}_{n \kappa} e^{-2 \nu_{1} \alpha r}\left(1-e^{-2 \alpha r}\right)^{\kappa^{\prime}+1}{ }_{2} F_{1}\left(-n, n+2\left(\nu_{1}+\kappa^{\prime}+1\right) ; 1+2 \nu_{1} ; e^{-2 \alpha r}\right),
$$

and

$$
\begin{gathered}
G_{n \kappa}(r)=\frac{\mathcal{N}_{n \kappa}}{M+E_{n \kappa}-C_{s}}\left(\frac{2 \alpha\left(\kappa^{\prime}+1\right) e^{-2 \alpha r}}{1-e^{-2 \alpha r}}-2 \alpha \nu_{1}+\frac{\kappa^{\prime}}{r}\right) F_{n \kappa}(r) \\
+\mathcal{N}_{n \kappa} \frac{2 n \alpha\left(n+2 \nu_{1}+2 \kappa^{\prime}+2\right)}{\left(M+E_{n \kappa}-C_{s}\right)\left(1+2 \nu_{1}\right)}\left(1-e^{-2 \alpha r}\right)^{\kappa^{\prime}+1}\left(e^{-2 \alpha r}\right)^{\nu_{1}+1} \\
\quad \times{ }_{2} F_{1}\left(-n+1 ; n+2\left(\nu_{1}+\kappa^{\prime}+1\right)+1 ; 2\left(1+\nu_{1}\right) ; e^{-2 \alpha r}\right) .
\end{gathered}
$$


respectively. In view of pseudospin symmetry, we obtain the energy spectrum from Eq. (46) by making the change $\kappa \rightarrow \kappa^{\prime \prime}$ as

$$
\sqrt{\left(M+E_{n \kappa}\right)\left(M-E_{n \kappa}+C_{p s}\right)}+\alpha\left(n+\kappa^{\prime \prime}\right)=\frac{\left(E_{n \kappa}-M-C_{p s}\right) A}{2\left(n+\kappa^{\prime \prime}\right)}, \kappa^{\prime \prime}=-n,
$$

where

$$
\kappa^{\prime \prime}=\frac{1}{2}+\frac{1}{2} \sqrt{(2 \kappa-1)^{2}+4 D\left(E_{n \kappa}-M-C_{p s}\right)} .
$$

The lower and upper wave functions can be obtained as follows

$$
G_{n \kappa}(r)=\mathcal{N}_{n \kappa} e^{-2 \nu_{2} \alpha r}\left(1-e^{-2 \alpha r}\right)^{\kappa^{\prime \prime}}{ }_{2} F_{1}\left(-n, n+2\left(\nu_{2}+\kappa^{\prime \prime}\right) ; 1+2 \nu_{2} ; e^{-2 \alpha r}\right),
$$

and

$$
\begin{gathered}
F_{n \kappa}(r)=\frac{\mathcal{N}_{n \kappa}}{\left(M-E_{n \kappa}+C_{p s}\right)}\left(\frac{2 \alpha \kappa^{\prime \prime} e^{-2 \alpha r}}{1-e^{-2 \alpha r}}-2 \alpha \nu_{2}-\frac{\kappa^{\prime \prime}}{r}\right) G_{n \kappa}(r) \\
+\mathcal{N}_{n \kappa} \frac{2 n \alpha\left(n+2 \nu_{2}+2 \kappa^{\prime \prime}\right)}{\left(M-E_{n \kappa}+C_{p s}\right)\left(1+2 \nu_{2}\right)}\left(1-e^{-2 \alpha r}\right)^{\kappa^{\prime \prime}}\left(e^{-2 \alpha r}\right)^{\nu_{2}+1} \\
\quad \times{ }_{2} F_{1}\left(-n+1 ; n+2\left(\nu_{2}+\kappa^{\prime \prime}\right)+1 ; 2\left(1+\nu_{2}\right) ; e^{-2 \alpha r}\right) .
\end{gathered}
$$

Fifth, the exact Dirac-Coulomb problem $(\alpha=0)$ has the following energy equations:[1]

$$
4 N_{p s}^{2}\left(M+E_{n \kappa}\right)=\left(M-E_{n \kappa}\right) A^{2}, N_{p s}=n+\kappa, n=0,1,2, \cdots
$$

and

$$
4 N_{s}^{2}\left(M-E_{n \kappa}\right)=A^{2}\left(M+E_{n \kappa}\right), N_{s}=n+\kappa+1, n=0,1,2, \cdots,
$$

in the limitation of the exact pseudospin $\left(C_{p s}=0\right)$ and spin $\left(C_{s}=0\right)$ symmetry, respectively. Obviously, in making the following changes $E_{n \kappa} \rightarrow-E_{n \kappa}$ and $\kappa \rightarrow \kappa-1$, one can easily switch off from spin symmetry, Eq. (76), into pseudospin symmetry, Eq. (75). Furthermore, Eqs. (75) and (76) are identical to Eqs. (37) and (47) of Ref. [48] (if one sets $A=0=C$ and $B \rightarrow A$ ). They are also identical to Eqs. (40) and (52) of Ref. [49].

\section{NUMERICAL RESULTS}

From Eq. (35), for small potential strength parameter $A=\sqrt{2}$, we calculate some numerical values of the bound state energies of the Schrödinger equation with the Yukawa

[1] The bound state solutions are exact for the case $\alpha=0$ since it lies in the short screening range. 
potential for various values of quantum numbers $n, l$ and screening parameter $\alpha(\hbar=\mu=1)$. Our approximated results in Table 3 are compared with those of $[16,17,19]$ together with the results of [13] who solved the Schrödinger equation numerically. Our results are in good agreement for small $\alpha$ values and becomes worse as $\alpha$ increases since the approximation used to substitute the singular part of Yukawa potential, $r^{-1}$ and the centrifugal term $r^{-2}$ are true for $\alpha r \ll 1$. As the potential strength parameter $A$ becomes larger, the numerical solution of the Schrödinger equation is extremely difficult because the screened Coulomb (Yukawa) potential is very deep and the wavefunction becomes very sharply peaked near the origin. Because of the instability of the wave function the energy eigenvalues become fairly inaccurate as the strength $A$ increases.

Based on the spin symmetry case, from Eq. (28), we can calculate some relativistic particle $E_{n \kappa}^{+}$and antiparticle $E_{n \kappa}^{-}$bound state energies with values of parameters $\alpha=0.1$ $\mathrm{fm}^{-1}, A=1.0, M=5.0 \mathrm{fm}^{-1}$ and $C_{s}=4.9 \mathrm{fm}^{-1}$ for various states with quantum numbers $n$ and $l(\kappa<0, \kappa>0)$ in the units $\hbar=c=1$ are used. We present our results in Table 4. Hence, one can see that there are degenerate eigenvalues of the spin partners within the attractive scalar and repulsive vector Yukawa potentials. For example, the Dirac spin doublet eigenstate partners are: $\left(1 s_{1 / 2}, 0 p_{3 / 2}, 2 p_{3 / 2}, 1 d_{5 / 2}, 3 d_{5 / 2}, 2 f_{7 / 2}, 4 f_{7 / 2}, 3 g_{9 / 2}, 4 h_{11 / 2}\right)$, $\left(2 s_{1 / 2}, 3 p_{3 / 2}, 0 d_{5 / 2}, 4 d_{5 / 2}, 1 f_{7 / 2}, 2 g_{9 / 2}, 3 h_{11 / 2}\right)$, and $\left(3 s_{1 / 2}, 4 p_{3 / 2}, 0 f_{7 / 2}, 1 g_{9 / 2}, 2 h_{11 / 2}, 1 p_{1 / 2}, 0 d_{3 / 2}\right) \ldots$ etc.

Using Eq. (46), we can also calculate some relativistic particle $E_{n \kappa}^{+}$and antiparticle $E_{n \kappa}^{-}$pseudospin symmetric bound state energies with values of parameters $\alpha=0.1 \mathrm{fm}^{-1}$, $A=1.0, M=5.0 \mathrm{fm}^{-1}$ and $C_{p s}=-5.0 \mathrm{fm}^{-1}$ for various states with quantum numbers $n$ and $\widetilde{l}(\kappa<0, \kappa>0)$ in the units $\hbar=c=1$. We present our results in Table 5 . One can see that there are degenerate eigenvalues of the pseudospin partners within the attractive scalar and repulsive vector Yukawa potentials. As an example, the Dirac pseudospin doublet eigenstate partners are: $\left(1 p_{3 / 2}, 2 s_{1 / 2}, 2 d_{5 / 2}\right),\left(1 d_{5 / 2}, 2 f_{7 / 2}, 0 d_{3 / 2}\right),\left(1 f_{7 / 2}, 2 g_{9 / 2}, 0 f_{5 / 2}, 1 d_{3 / 2}\right)$, $\left(1 g_{9 / 2}, 2 h_{11 / 2}, 0 g_{7 / 2}, 1 f_{5 / 2}\right)$, and $\left(1 h_{11 / 2}, 0 h_{9 / 2}, 1 g_{7 / 2}\right), \ldots$ etc. When $n=1$, there is a finite number of bound states where $1 \leq \widetilde{l} \leq 25(\kappa=-25, \kappa=24)$ and when $n=2,1 \leq \widetilde{l} \leq 26$ $(\kappa=-26, \kappa=23)$. 


\section{CONCLUSION AND OUTLOOK}

We have investigated the approximate bound state solutions of the Dirac equation for the screened Coulomb potential model with an arbitrary spin-orbit $\kappa$ - state under the conditions of the spin (pseudospin) symmetry limitation in the framework of the shortcut of the NU method including the usual approximation scheme to approximate the centrifugal (pseudocentrifugal) barrier term. By setting $\Sigma(r)(\Delta(r))$ to the spherically symmetric screened Coulomb potential model, we have derived the Dirac bound state energy spectra and associated two-component spinor wave functions for arbitrary spin-orbit $\kappa$ state that provides an approximate solution to the spin- and pseudo-spin symmetry. We have also discussed in detail how to choose the appropriate physical values for the spin constant $C_{s}$ from Eq. (25). In the presence of spin symmetry, we find that the appropriate value for $C_{s}$ falls in the range $-20 \mathrm{fm}^{-1} \leq C_{s} \leq 8.8 \mathrm{fm}^{-1}$ for positive energy part while $0 \mathrm{fm}^{-1}<C_{s} \leq 9.6 \mathrm{fm}^{-1}$ for the negative energy part. Thus, the allowed values for the $C_{s}$ in considering the whole spectrum (particle and antiparticle) falls in $4.9 \mathrm{fm}^{-1}<C_{s} \leq 8.8 \mathrm{fm}^{-1}$ after neglecting the negative part of energy according to the requirement $E_{n, \kappa} \neq-M$. However, in the presence of pseudosymmetry, we find $C_{p s}$ value falls in the range $-8 \mathrm{fm}^{-1} \leq C_{p s} \leq 20 \mathrm{fm}^{-1}$ in the antiparticle energy spectrum demanding that $E_{n, \kappa} \neq M$. The Yukawa interaction between an electron and heavy nucleus appears to be less attractive as the value of $\alpha$ increases. The resulting solutions of the wave functions are being expressed in terms of the Jacobi polynomials (or hypergeometric functions). We have shown that the present spin symmetry can be easily reduced to the non-relativistic solution when one inserts $S(r)=+V(r)$ (i.e., $\Delta(r)=0$ or $\left.C_{s}=0\right)$. The non-relativistic limits of our solution are obtained by imposing appropriate changes of parameters $\kappa(\kappa+1) \rightarrow l(l+1)$ in the spin symmetry limits. Furthermore, when $\alpha \rightarrow 0$, our results can be reduced to the well-known bound state solutions for the Coulomb potential model. We must point out that the numerical calculations for eigenenergies of the Dirac states involved in Eqs. (28) and (46) are sensitive to the choice of the parameters $C_{s}$, $C_{p s}, \alpha, A$ and $M$. The spin (pseudospin) limit Dirac energy spectrum computed in Table 3 (Table 4) for arbitrarily chosen set of parameters are in the form of valence (hole) states. In order to remove the extra degeneracies in energy levels, we have solved Dirac-Yukawa problem in the presence of spin and pseudospin symmetry by adding a centrifugal-like term, i.e., $V(r)=-A e^{-\alpha r} / r+D / r^{2}$. 
Finally, the solution of the Dirac-Coulomb problem can be readily obtained from our solutions by setting $\alpha=0$. Hence, we can obtain expressions for the exact energy eigenvalues for the exact spin and pseudospin limitations. These exact solutions are identical to the ones found recently in Refs. [48,49].

\section{Acknowledgments}

The author thanks the two kind referees for their enlightening suggestions which helped him to improve this work.

\section{Appendix A: Résumé of Parametric Generalization of NU Method}

We present the Nikiforov-Uvarov essential polynomials, root, eigenvalues and wave functions expressed in terms of the constants $c_{i}(i=1,2, \cdots, 13)$ and $\xi_{j}(j=0,1,2)$ :

(i) Constants:

$$
\begin{gathered}
c_{4}=\frac{1}{2}\left(1-c_{1}\right), c_{5}=\frac{1}{2}\left(c_{2}-2 c_{3}\right), c_{6}=c_{5}^{2}+\xi_{2}, \\
c_{7}=2 c_{4} c_{5}-\xi_{1}, c_{8}=c_{4}^{2}+\xi_{0}, c_{9}=c_{3}\left(c_{7}+c_{3} c_{8}\right)+c_{6}, \\
c_{10}=c_{1}+2 c_{4}+2 \sqrt{c_{8}}-1>-1, c_{11}=1-c_{1}-2 c_{4}+\frac{2}{c_{3}} \sqrt{c_{9}}>-1, \\
c_{12}=c_{4}+\sqrt{c_{8}}>0, c_{13}=-c_{4}+\frac{1}{c_{3}}\left(\sqrt{c_{9}}-c_{5}\right)>0, c_{3} \neq 0 .
\end{gathered}
$$

(ii) Polynomials:

$$
\begin{gathered}
\pi(x)=c_{4}+\sqrt{c_{8}}+c_{5} x-\left(\sqrt{c_{9}}+c_{3} \sqrt{c_{8}}\right) x, \\
k=-\left(c_{7}+2 c_{3} c_{8}\right)-2 \sqrt{c_{8} c_{9}}, \\
\tau(x)=1+2 \sqrt{c_{8}}-\left[c_{2}+2\left(\sqrt{c_{9}}+c_{3} \sqrt{c_{8}}-c_{5}\right)\right] x, \\
\tau^{\prime}(x)=-2 c_{3}-2\left(\sqrt{c_{9}}+c_{3} \sqrt{c_{8}}\right)<0 .
\end{gathered}
$$

(iii) Energy equation:

$$
c_{2} n-(2 n+1) c_{5}+(2 n+1)\left(\sqrt{c_{9}}+c_{3} \sqrt{c_{8}}\right)+n(n-1) c_{3}+c_{7}+2 c_{3} c_{8}+2 \sqrt{c_{8} c_{9}}=0 .
$$

(iv) Wave functions:

$$
\rho(x)=x^{c_{10}}\left(1-c_{3} x\right)^{c_{11}},
$$




$$
\begin{gathered}
\phi(x)=x^{c_{12}}\left(1-c_{3} x\right)^{c_{13}}, c_{12}>0, c_{13}>0, \\
y_{n}(x)=P_{n}^{\left(c_{10}, c_{11}\right)}\left(1-2 c_{3} x\right), c_{10}>-1, c_{11}>-1, \\
F_{n \kappa}(x)=\mathcal{N}_{n \kappa} x^{c_{12}}\left(1-c_{3} x\right)^{c_{13}} P_{n}^{\left(c_{10}, c_{11}\right)}\left(1-2 c_{3} x\right), \\
=\mathcal{N}_{n \kappa} x^{c_{12}}\left(1-c_{3} x\right)^{c_{13}}{ }_{2} F_{1}\left(-n, 1+c_{10}+c_{11}+n ; c_{10}+1 ; c_{3} x\right), x \in\left[0,1 / c_{3}\right], c_{3} \neq 0 .
\end{gathered}
$$

where $\mathcal{N}_{n \kappa}$ is a normalization constants. Further, $P_{n}^{(\mu, \nu)}(x), \mu>-1, \nu>-1$ and $x \in[-1,1]$ are the Jacobi polynomials with

$$
P_{n}^{(\alpha, \beta)}(1-2 s)=\frac{(\alpha+1)_{n}}{n !}{ }_{2} F_{1}(-n, 1+\alpha+\beta+n ; \alpha+1 ; s) .
$$

\section{Appendix B: Calculations of the Normalization Constants}

The normalization constant, $\mathcal{N}_{n l}$ can be determined in closed form. We start by using the relation between the hypergeometric function and the Jacobi polynomials (see formula $(8.962 .1)$ in $[45])$ :

$$
\begin{gathered}
{ }_{2} F_{1}\left(-n, n+\nu+\mu+1 ; \nu+1 ; \frac{1-x}{2}\right)=\frac{n !}{(\nu+1)_{n}} P_{n}^{(\nu, \mu)}(x), \\
(\nu+1)_{n}=\frac{\Gamma(n+\nu+1)}{\Gamma(\nu+1)}
\end{gathered}
$$

to rewrite the wave functions in $(32)$ as

$$
F_{n \kappa}(r)=\mathcal{N}_{n \kappa} \frac{n ! \Gamma\left(2 \nu_{1}+1\right)}{\Gamma\left(n+2 \nu_{1}+1\right)} e^{-2 \nu_{1} \alpha r}\left(1-e^{-2 \alpha r}\right)^{\kappa+1} P_{n}^{\left(2 \nu_{1}, 2 \kappa+1\right)}\left(1-2 e^{-2 \alpha r}\right) .
$$

From the normalization condition $\int_{0}^{\infty}\left[u_{n, l}(r)\right]^{2} d r=1$ and under the coordinate change $x=1-2 e^{-2 \alpha r}$, the normalization constant in (B2) is given by

$$
\mathcal{N}_{n \kappa}^{-2}=\frac{1}{\alpha}\left[\frac{n ! \Gamma\left(2 \nu_{1}+1\right)}{\Gamma\left(n+2 \nu_{1}+1\right)}\right]^{2} \int_{-1}^{1}\left(\frac{1-x}{2}\right)^{2 \nu_{1}}\left(\frac{1+x}{2}\right)^{2 \kappa+1}\left(\frac{1+x}{2}\right)\left[P_{n}^{\left(2 \nu_{1}, 2 \kappa+1\right)}(x)\right]^{2} d x .
$$

The calculation of this integral can be done by writing

$$
\frac{1+x}{2}=1-\left(\frac{1-x}{2}\right)
$$


and using the following two integrals (see formula (7.391.5) in [45]):

$$
\int_{-1}^{1}(1-x)^{\nu-1}(1+x)^{\mu}\left[P_{n}^{(\nu, \mu)}(x)\right]^{2} d x=2^{\nu+\mu} \frac{\Gamma(n+\nu+1) \Gamma(n+\mu+1)}{n ! \nu \Gamma(n+\nu+\mu+1)},
$$

which is valid for $\operatorname{Re}(\nu)>0$ and $\operatorname{Re}(\mu)>-1$ and (see formula (7.391.1) in [45]):

$$
\int_{-1}^{1}(1-x)^{\nu}(1+x)^{\mu}\left[P_{n}^{(\nu, \mu)}(x)\right]^{2} d x=2^{\nu+\mu+1} \frac{\Gamma(n+\nu+1) \Gamma(n+\mu+1)}{n ! \Gamma(n+\nu+\mu+1)(2 n+\nu+\mu+1)},
$$

which is valid for $\operatorname{Re}(\nu)>-1, \operatorname{Re}(\mu)>-1$. Finally, we have carried out relativistic and non-relativistic normalization constants as

$$
\mathcal{N}_{n \kappa}=\frac{1}{\Gamma\left(2 \nu_{1}+1\right)}\left[\frac{\alpha \nu_{1}\left(n+\nu_{1}+\kappa+1\right)}{(n+\kappa+1)} \frac{\Gamma\left(n+2 \nu_{1}+1\right) \Gamma\left(n+2 \nu_{1}+2 \kappa+2\right)}{n ! \Gamma(n+2 \kappa+2)}\right]^{1 / 2},
$$

and

$$
\mathcal{N}_{n \kappa}=\frac{1}{\Gamma\left(2 \varepsilon_{n l}+1\right)}\left[\frac{\alpha \varepsilon_{n l}\left(n+l+\varepsilon_{n l}+1\right)}{(n+l+1)} \frac{\Gamma\left(n+2 \varepsilon_{n l}+1\right) \Gamma\left(n+2 \varepsilon_{n l}+2 l+2\right)}{n ! \Gamma(n+2 l+2)}\right]^{1 / 2} .
$$

respectively. 
[1] H. Yukawa, Proc. Phys. Math. Soc. 17 (1935) 48; H. Yukawa, Proc. Phys. Math. Soc. 19 (1937) 1084 .

[2] R. Messina and H. Lowen, Phys. Rev. Lett. 91 (2003) 146101.

[3] S. Kar and Y.K. Ho, Phys. Rev. A 75 (2007) 062509.

[4] S.A. Khrapak, A.V. Ivlev, G.E. Morfill, S.K. Zhdanov and H.M. Thomas, IEEE Transactions on Plasma Science 32 (2004) 555.

[5] P.J. Siemens, Phys. Rev. C 1 (1970) 98.

[6] S.J. Lee, H.H. Gan, E.D. Cooper and S. Das Gupta, Phys. Rev. C 40 (1989) 2585.

[7] W. Greiner, Relativistic Quantum Mechanics: Wave Equations, 3rd edn. (Springer, Berlin, 2000).

[8] M.A. Preston and R.K. Bhaduri, Structure of the Nucleus (Addison-Wesley, Reading, 1975).

[9] W. Frank, Nature 445 (2007) 156.

[10] L.I. Schiff, Quantum Mechanics, 3rd edn. (McGraw-Hill, Singapore, 1968), p.325.

[11] O.A. Gomes, H. Chacham and J.R. Mohallem, Phys. Rev. 50 (1994) 228.

[12] R. Dutt, A. Ray and P.P. Ray, Phys. Lett. A 83 (1981) 65; D. Singh and Y.P. Varshni, Phys. Rev. A 28 (1983) 2606; H. de Meyer et al, J. Phys. A 18 (1985) 849.

[13] F.J. Rogers, H.C. Jr Graboske and D.G. Hardwood, Phys. Rev. A 1 (1970) 1577.

[14] C.S. Lai, Phys. Rev. A 26 (1982) 2245.

[15] R. Dutt et al, J. Phys. A18 (1985) 1379; C.S. Lam and Y.P. Varshni, Phys. Rev. A 6 (1972) 1391.

[16] A. Chatterjee, J. Phys. A: Math. Gen. 19 (1986) 3707.

[17] C. Lee, Phys. Lett. A 267 (2000) 101.

[18] A.D. Alhaidari, H. Bahlouli and M.S. Abdelmonem, J. Phys. A: Math. Theor. 41 (2008) 032001.

[19] M. Grant and C.S. Lai, Phys. Rev. A 20 (1979) 718.

[20] J.D. Hirschfelder, J. Chem. Phys. 33 (1960) 1462 ; J. Killingbeck, Phy. Lett. A 65 (1987) 87.

[21] B. Gönül, K. Köksal and E. Bakır, Phys. Scr. 73 (2006) 279.

[22] S.M. Ikhdair and R. Sever, J. Math. Chem. 41 (4) (2007) 329, 343.

[23] M. Karakoc and I. Boztosun, Int. J. Mod. Phys. E 15 (6) (2006) 1253. 
[24] J. Broeckhove, F. Arickx, W. Vanroose and V.S. Vasilevsky, J. Phys. A: Math. Gen. 37 (2004) 7769 .

[25] J. Aguilar and J.M. Combes, Commun. Math. Phys. 22 (1971) 269; Y.K. Ho, Phys. Rep. 99 (1983) 1.

[26] T.Y. Wu and W.Y. Pauchy Hwang, Relativistic Quantum Mechanics and Quantum Fields (World Scientific, Singapore, 1991).

[27] J.N. Ginocchio, Phys. Rep. 414 (2005) 165.

[28] P.R. Page, T. Goldman and J.N. Ginocchio, Phys. Rev. Lett. 86 (2001) 204.

[29] A. Arima, M. Harvey and K. Shimizu, Phys. Lett. B 30 (1969) 517.

[30] K.T. Hecht and A. Adler, Nucl. Phys. A 137 (1969) 129.

[31] J.N. Ginocchio and D.G. Madland, Phys. Rev. C 57 (1998) 1167.

[32] A. Bohr, I. Hamarnoto and B.R. Motelson, Phys. Scr. 26 (1982) 267.

[33] J. Dudek, W. Nazarewicz, Z. Szymanski and G.A. Leander, Phys. Rev. Lett. 59 (1987) 1405.

[34] D. Troltenier, C. bahri and J. P. Draayer, Nucl. Phys. A 586 (1995) 53.

[35] S.M. Ikhdair, J. Math. Phys. 52 (2011) 052303; S.M. Ikhdair and R. Sever, J. Math. Phys. 52 (2011); doi:10.1063/1.3671640.

[36] S.M. Ikhdair and R. Sever, J. Phys. A: Math. Theor. 44 (2011) 355301; S.M. Ikhdair, C. Berkdemir and R. Sever, Appl. Math. Comput. 217 (22) (2011) 9019.

[37] S.M. Ikhdair, J. Math. Phys. 51 (2010) 023525.

[38] F. Dominguez-Adame and A. Rodriguez, Phys. Lett. A 198 (1995) 275.

[39] A.F. Nikiforov and V.B. Uvarov, Special Functions of Mathematical Physics (Birkhäuser: Basel, 1988).

[40] S.M. Ikhdair and R. Sever, Cent. Eur. J. Phys. 8 (2010) 652.

[41] M.R. Setare and S. Haidari, Phys. Scr. 81 (2010) 065201.

[42] R.L. Greene and C. Aldrich, Phys. Rev. A 14 (1976) 2363.

[43] S.M. Ikhdair, Chem. Phys. 361 (2009) 9; S.M. Ikhdair, J. Quantum Infor. Science 1 (2011) 73.

[44] A.D. Alhaidari, H. Bahlouli and A. Al-Hasan, Phys. Lett. A 349 (2006) 87.

[45] M. Abramowitz and I.A. Stegun, Handbook of Mathematical Functions (Dover Publication, New York, 1972).

[46] I.S. Gradshteyn and I.M. Ryzhik, Tables and integrals, series and products (New York, Academic, 1969). 
[47] C. Berkdemir and Y.-F. Cheng, Phys. Scr. 79 (2009) 035003.

[48] M. Hamzavi, A.A. Rajabi and H. Hassanabadi, Few-Body Syst. 48 (2010) 171.

[49] O. Aydoğdu and R. Sever, Ann. Phys.325 (2010) 373. 
FIG. 1: Spin symmetry Dirac ground valence state energy level for (a) a particle and (b) antiparticle of mass $M=5.0 \mathrm{fm}^{-1}$ as a function of the coupling constant $A$ for several values of the range of the screened Coulomb potential.

FIG. 2: Dirac ground valence state energy level for (a) a particle and (b) anti-particle of mass $M=5.0 \mathrm{fm}^{-1}$ as a function of the spin symmetry constant $C_{s}$ for several values of the spin-orbit $\kappa$.

FIG. 4: Pseudospin symmetry Dirac ground hole state energy level for (a) a particle and (b) antiparticle of mass $M=5.0 \mathrm{fm}^{-1}$ as a function of the coupling constant $A$ for several values of the range of the screened Coulomb potential.

FIG. 5: Dirac ground hole state energy level for (a) a particle and (b) anti-particle of mass $M=5.0$ $\mathrm{fm}^{-1}$ as a function of the pseudospin symmetry constant $C_{p s}$ for several values of the spin-orbit $\kappa$.

FIG. 3: Spin symmetry Dirac upper and lower spinor wave functions of ground $0 p_{1 / 2}$ and first excited $1 p_{1 / 2}$ states of (a) particle and (b) antiparticle with $\kappa=1$. 
FIG. 6: Pseudospin symmetry Dirac upper and lower spinor wave functions of ground $0 d_{3 / 2}$ and first excited $1 d_{3 / 2}$ states of (a) particle and (b) antiparticle with $\kappa=2$.

TABLE I: Specific values of the constants in Dirac-Yukawa problem.

\begin{tabular}{ll}
\hline Spin symmetry case & Pseudospin symmetry case \\
\hline$c_{1}=1$ & $c_{1}=1$ \\
$c_{2}=1$ & $c_{2}=1$ \\
$c_{3}=1$ & $c_{3}=1$ \\
$c_{4}=0$ & $c_{4}=0$ \\
$c_{5}=-\frac{1}{2}$ & $c_{5}=-\frac{1}{2}$ \\
$c_{6}=\frac{1}{4}\left[1+4\left(\nu_{1}^{2}+\omega_{1}\right)\right]$ & $c_{6}=\frac{1}{4}\left[1+4\left(\nu_{2}^{2}+\omega_{2}\right)\right]$ \\
$c_{7}=-2 \nu_{1}^{2}-\omega_{1}+\kappa(\kappa+1)$ & $c_{7}=-2 \nu_{2}^{2}-\omega_{2}+\kappa(\kappa-1)$ \\
$c_{8}=\nu_{1}^{2}$ & $c_{8}=\nu_{2}^{2}$ \\
$c_{9}=\frac{1}{4}(2 \kappa+1)^{2}$ & $c_{9}=\frac{1}{4}(2 \kappa-1)^{2}$ \\
$c_{10}=2 \nu_{1}$ & $c_{10}=2 \nu_{2}$ \\
$c_{11}=2 \kappa+1$ & $c_{11}=2 \kappa-1$ \\
$c_{12}=\nu_{1}$ & $c_{12}=\nu_{2}$ \\
$c_{13}=\kappa+1$ & $c_{13}=\kappa$ \\
$\xi_{2}=\nu_{1}^{2}+\omega_{1}$ & $\xi_{2}=\nu_{2}^{2}+\omega_{2}$ \\
$\xi_{1}=2 \nu_{1}^{2}+\omega_{1}-\kappa(\kappa+1)$ & $\xi_{1}=2 \nu_{2}^{2}+\omega_{2}-\kappa(\kappa-1)$ \\
$\xi_{0}=\nu_{1}^{2}$ & $\xi_{0}=\nu_{2}^{2}$ \\
\hline
\end{tabular}


TABLE II: Spin symmetric Dirac-Yukawa ground valence energy spectrum (in units of $\mathrm{fm}^{-1}$ ) of the particle and antiparticle as a function of $C_{s}$ for various values of $\kappa$.

\begin{tabular}{|c|c|c|c|c|c|c|c|c|c|}
\hline$C_{s}$ & $E_{0, \kappa=1}^{+}$ & $E_{0, \kappa=3}^{+}$ & $E_{0, \kappa=5}^{+}$ & $E_{0, \kappa=7}^{+}$ & $E_{0, \kappa=9}^{-}$ & $C_{s}$ & $E_{0, \kappa=1}^{-}$ & $E_{0, \kappa=3}^{-}$ & $E_{0, \kappa=5}^{-}$ \\
\hline-20 & 3.328 & 4.632 & 4.880 & 4.962 & 4.992 & -20 & -24.999 & -24.995 & -24.988 \\
\hline-15 & 3.622 & 4.707 & 4.913 & 4.977 & 4.998 & -15 & -19.998 & -19.994 & -19.986 \\
\hline-10 & 3.916 & 4.783 & 4.943 & 4.990 & 5.000 & -10 & -14.998 & -14.992 & -14.982 \\
\hline-5 & 4.209 & 4.857 & 4.972 & 4.999 & 4.996 & -5 & -9.997 & -9.989 & -9.976 \\
\hline 0 & 4.502 & 4.929 & 4.995 & 4.997 & 4.975 & 0 & -4.996 & -4.984 & -4.964 \\
\hline 5 & 4.792 & 4.990 & 4.993 & 4.951 & 4.883 & 1 & -3.996 & -3.982 & -3.960 \\
\hline 6 & 4.849 & 4.998 & 4.982 & 4.921 & 4.829 & 4 & -0.993 & -0.974 & -0.940 \\
\hline 7 & 4.905 & 5.000 & 4.958 & 4.865 & 4.726 & 4.8 & -0.192 & -0.170 & -0.131 \\
\hline 8 & 4.957 & 4.988 & 4.897 & 4.722 & 4.363 & 4.9 & -0.092 & -0.069 & -0.030 \\
\hline 8.5 & 4.980 & 4.968 & 4.818 & - & - & 5 & 0.007856 & 0.032 & 0.072 \\
\hline 8.8 & 4.992 & 4.942 & 4.689 & - & - & 6 & 1.010 & 1.039 & 1.090 \\
\hline 9 & 4.998 & 4.910 & - & - & - & 7 & 2.013 & 2.053 & 2.121 \\
\hline 9.5 & 4.987 & - & - & - & - & 8 & 3.019 & 3.079 & 3.188 \\
\hline 10 & - & - & - & - & - & 9 & 4.038 & 4.173 & - \\
\hline 10.5 & - & - & - & - & - & 9.5 & 4.577 & - & - \\
\hline 10.6 & 5.498 & - & - & - & - & 9.6 & 4.702 & - & - \\
\hline 10.7 & 5.623 & - & - & - & - & 9.7 & - & - & - \\
\hline 10.8 & 5.737 & - & - & - & - & 10.5 & - & - & - \\
\hline 10.9 & 5.846 & - & - & - & - & 10.6 & 5.232 & - & - \\
\hline 11 & 5.953 & 5.754 & - & - & - & 11 & 5.200 & 5.360 & - \\
\hline 11.1 & 6.058 & 5.899 & - & - & - & 11.3 & 5.205 & 5.272 & - \\
\hline 11.3 & 6.266 & 6.147 & - & - & - & 11.4 & 5.208 & 5.258 & 5.511 \\
\hline 11.4 & 6.368 & 6.262 & 5.998 & - & - & 11.5 & 5.212 & 5.247 & 5.450 \\
\hline 12 & 6.979 & 6.912 & 6.786 & 6.562 & - & 12 & 5.233 & 5.218 & 5.327 \\
\hline 13 & 7.986 & 7.944 & 7.870 & 7.759 & 7.600 & 15 & 5.396 & 5.208 & 5.208 \\
\hline 14 & 8.990 & 8.959 & 8.905 & 8.828 & 8.724 & 20 & 5.686 & 5.268 & 5.205 \\
\hline 15 & 9.992 & 9.967 & 9.925 & 9.866 & 9.787 & & & & \\
\hline 20 & 14.996 & 14.984 & 14.964 & 14.935 & 14.898 & & & & \\
\hline
\end{tabular}


TABLE III: Nonrelativistic bound state energies of the Yukawa potential in units of $\hbar=m=1$. For comparison, we set $A=\sqrt{2}$ and $\alpha=g A$.

\begin{tabular}{|c|c|c|c|c|c|c|c|}
\hline Stat & $n l g$ & NU (Present) & [13] (Numerical) & [23] (AIM) & [21] (SUSY) & {$[16]$} & {$[17]$} \\
\hline \multirow[t]{6}{*}{$1 s$} & $\begin{array}{lll}0 & 0 & 0.002\end{array}$ & -0.996004 & -0.9960 & -0.996006 & -0.99601 & -0.99601 & -0.9960 \\
\hline & 0.005 & -0.990025 & -0.9900 & -0.990037 & -0.99004 & -0.99004 & - \\
\hline & 0.01 & -0.9801 & -0.9801 & -0.980149 & -0.98015 & -0.98015 & -0.9801 \\
\hline & 0.02 & -0.9604 & -0.9606 & -0.960592 & -0.96059 & -0.96059 & -0.9606 \\
\hline & 0.025 & -0.950625 & -0.9509 & -0.950922 & -0.95092 & -0.95092 & - \\
\hline & 0.05 & -0.9025 & -0.9036 & -0.903632 & -0.90363 & -0.90363 & -0.9036 \\
\hline \multirow[t]{6}{*}{$2 \mathrm{~s}$} & 100.002 & -0.246016 & -0.2460 & -0.246023 & -0.24602 & -0.24602 & -0.2460 \\
\hline & 0.005 & -0.2401 & -0.2401 & -0.240148 & -0.24015 & -0.24015 & - \\
\hline & 0.01 & -0.2304 & -0.2306 & -0.230586 & -0.23059 & -0.23058 & -0.2306 \\
\hline & 0.02 & -0.2116 & -0.21230 & -0.212296 & -0.21230 & -0.21229 & -0.2124 \\
\hline & 0.025 & -0.2025 & -0.2036 & -0.203551 & -0.20355 & -0.20352 & - \\
\hline & 0.05 & -0.16 & -0.1635 & -0.163542 & -0.16351 & -0.16325 & -0.1650 \\
\hline \multirow[t]{6}{*}{$2 \mathrm{p}$} & $\begin{array}{lll}0 & 1 & 0.002\end{array}$ & -0.246016 & -0.2460 & -0.246019 & -0.24602 & -0.24602 & -0.2460 \\
\hline & 0.005 & -0.2401 & -0.2401 & -0.240123 & -0.24012 & -0.2412 & - \\
\hline & 0.01 & -0.2304 & -0.2305 & -0.230490 & -0.23049 & -0.23049 & -0.2305 \\
\hline & 0.02 & -0.2116 & -0.2119 & -0.211926 & -0.21192 & -0.21193 & -0.2120 \\
\hline & 0.025 & -0.2025 & -0.2030 & -0.202984 & -0.20299 & -0.20299 & - \\
\hline & 0.05 & -0.16 & -0.1615 & -0.161480 & -0.16144 & -0.16155 & -0.1625 \\
\hline \multirow[t]{6}{*}{$3 p$} & $\begin{array}{lll}1 & 10.002\end{array}$ & -0.107147 & -0.1072 & -0.107160 & -0.10716 & -0.10716 & -0.1072 \\
\hline & 0.005 & -0.101336 & -0.1014 & -0.101416 & -0.10142 & -0.10142 & - \\
\hline & 0.01 & -0.092011 & -0.09231 & -0.092306 & -0.09231 & -0.09236 & -0.09236 \\
\hline & 0.02 & -0.074711 & -0.07570 & -0.075704 & -0.07570 & -0.07563 & -0.07611 \\
\hline & 0.025 & -0.066736 & -0.06816 & -0.068157 & -0.06814 & -0.06799 & - \\
\hline & 0.05 & -0.033611 & -0.03712 & -0.037115 & -0.03739 & -0.03486 & -0.04236 \\
\hline \multirow[t]{5}{*}{$3 d$} & 020.002 & -0.107147 & -0.1072 & -0.107152 & -0.10715 & -0.10715 & -0.1072 \\
\hline & 0.005 & -0.101336 & -0.1014 & -0.101368 & -0.1014 & -0.10137 & - \\
\hline & 0.01 & -0.092011 & -0.09212 & -0.092122 & -0.09212 & -0.09212 & -0.09216 \\
\hline & 0.02 & -0.074711 & -0.07503 & -0.075030 & -0.07502 & -0.07504 & -0.07531 \\
\hline & 0.025 & -0.066736 & -0.06715 & -0.067146 & -0.06713 & -0.06718 & - \\
\hline
\end{tabular}


TABLE IV: Dirac valence bound state energies (in units of $\mathrm{fm}^{-1}$ ) of the spin symmetry Yukawa problem for various values of $n$ and $l$.

\begin{tabular}{|c|c|c|c|c|c|c|c|}
\hline \multicolumn{2}{|c|}{$l n, \kappa<0(l, j)$} & \multirow{2}{*}{$\frac{E_{n, \kappa<0}^{-}}{-}$} & \multirow{2}{*}{$\frac{E_{n, \kappa<0}^{+}}{-}$} & \multicolumn{2}{|c|}{$n, \kappa>0(l, j)$} & \multirow[t]{2}{*}{$E_{n, \kappa>0}^{-}$} & \multirow[t]{2}{*}{$E_{n, \kappa>0}^{+}$} \\
\hline $00,-1$ & $0 s_{1 / 2}$ & & & & & & \\
\hline $01,-1$ & $1 s_{1 / 2}$ & -0.098076 & 4.05808 & & & & \\
\hline $02,-1$ & $2 s_{1 / 2}$ & -0.0922956 & 4.78641 & & & & \\
\hline $03,-1$ & $3 s_{1 / 2}$ & -0.0826327 & 4.94209 & & & & \\
\hline $04,-1$ & $4 s_{1 / 2}$ & -0.0690436 & 4.98904 & & & & \\
\hline $10,-2$ & $0 p_{3 / 2}$ & -0.098076 & 4.05808 & 0,1 & $0 p_{1 / 2}$ & -0.0922956 & 4.78641 \\
\hline $11,-2$ & $1 p_{3 / 2}$ & - & - & 1,1 & $1 p_{1 / 2}$ & -0.0826327 & 4.94209 \\
\hline $12,-2$ & $2 p_{3 / 2}$ & -0.098076 & 4.05808 & 2,1 & $2 p_{1 / 2}$ & -0.0690436 & 4.98904 \\
\hline $13,-2$ & $3 p_{3 / 2}$ & -0.0922956 & 4.78641 & 3,1 & $3 p_{1 / 2}$ & -0.0514655 & 4.99998 \\
\hline $14,-2$ & $4 p_{3 / 2}$ & -0.0826327 & 4.94209 & 4,1 & $4 p_{1 / 2}$ & -0.0298154 & 4.99395 \\
\hline $20,-3$ & $0 d_{5 / 2}$ & -0.0922956 & 4.78641 & 0,2 & $0 d_{3 / 2}$ & -0.0826327 & 4.94209 \\
\hline $21,-3$ & $1 d_{5 / 2}$ & -0.098076 & 4.05808 & 1,2 & $1 d_{3 / 2}$ & -0.0690436 & 4.98904 \\
\hline $22,-3$ & $2 d_{5 / 2}$ & - & - & 2,2 & $2 d_{3 / 2}$ & -0.0514655 & 4.99998 \\
\hline $23,-3$ & $3 d_{5 / 2}$ & -0.098076 & 4.05808 & 3,2 & $3 d_{3 / 2}$ & -0.0298154 & 4.99395 \\
\hline $24,-3$ & $4 d_{5 / 2}$ & -0.0922956 & 4.78641 & 4,2 & $4 d_{3 / 2}$ & -0.0039874 & 4.97759 \\
\hline $30,-4$ & $0 f_{7 / 2}$ & -0.0826327 & 4.94209 & 0,3 & $0 f_{5 / 2}$ & -0.0690436 & 4.98904 \\
\hline $31,-4$ & $1 f_{7 / 2}$ & -0.0922956 & 4.78641 & 1,3 & $1 f_{5 / 2}$ & -0.0514655 & 4.99998 \\
\hline $32,-4$ & $2 f_{7 / 2}$ & -0.098076 & 4.05808 & 2,3 & $2 f_{5 / 2}$ & -0.0298154 & 4.99395 \\
\hline $33,-4$ & $3 f_{7 / 2}$ & - & - & 3,3 & $3 f_{5 / 2}$ & -0.0039874 & 4.97759 \\
\hline $34,-4$ & $4 f_{7 / 2}$ & -0.098076 & 4.05808 & 4,3 & $4 f_{5 / 2}$ & -0.0261492 & 4.95362 \\
\hline $40,-5$ & $0 g_{9 / 2}$ & -0.0690436 & 4.98904 & 0,4 & $0 g_{7 / 2}$ & -0.0514655 & 4.99998 \\
\hline $41,-5$ & $1 g_{9 / 2}$ & -0.0826327 & 4.94209 & 1,4 & $1 g_{7 / 2}$ & -0.0298154 & 4.99395 \\
\hline $42,-5$ & $2 g_{9 / 2}$ & -0.0922956 & 4.78641 & 2,4 & $2 g_{7 / 2}$ & -0.0039874 & 4.97759 \\
\hline $43,-5$ & $3 g_{9 / 2}$ & -0.098076 & 4.05808 & 3,4 & $3 g_{7 / 2}$ & -0.0261492 & 4.95362 \\
\hline $44,-5$ & $4 g_{9 / 2}$ & - & - & 4,4 & $4 g_{7 / 2}$ & -0.0607542 & 4.92325 \\
\hline $50,-6$ & $0 h_{11 / 2}$ & -0.0514655 & 4.99998 & 0,5 & $0 h_{9 / 2}$ & -0.0298154 & 4.99395 \\
\hline $51,-6$ & $1 h_{11 / 2}$ & -0.0690436 & 4.98904 & 1,5 & $1 h_{9 / 2}$ & -0.0039874 & 4.97759 \\
\hline $52,-6$ & $2 h_{11 / 2}$ & -0.0826327 & 4.94209 & 2,5 & $2 h_{9 / 2}$ & -0.0261492 & 4.95362 \\
\hline $53,-6$ & $3 h_{11 / 2}$ & -0.0922956 & $4.786\}_{3} 1$ & 3,5 & $3 h_{9}$ & -0.0607542 & 4.92325 \\
\hline
\end{tabular}


TABLE V: Dirac hole bound state energies (in units of $\mathrm{fm}^{-1}$ ) of the pseudospin symmetry Yukawa problem for various values of $n$ and $\tilde{l}$.

\begin{tabular}{|c|c|c|c|c|c|c|c|c|}
\hline$\widetilde{l}$ & $n, \kappa<0$ & $(l, j)$ & $E_{n, \kappa<0}^{-}$ & $E_{n, \kappa<0}^{+}$ & $n-1, \kappa>0$ & $(l+2, j+1)$ & $E_{n, \kappa>0}^{-}$ & $E_{n, \kappa>0}^{+}$ \\
\hline 1 & $1,-1$ & $1 s_{1 / 2}$ & - & - & 0,2 & $0 d_{3 / 2}$ & -4.603587 & -0.00817777 \\
\hline 2 & $1,-2$ & $1 p_{3 / 2}$ & -3.917958 & -0.00204188 & 0,3 & $0 f_{5 / 2}$ & -4.749130 & -0.01843870 \\
\hline 3 & $1,-3$ & $1 d_{5 / 2}$ & -4.603587 & -0.00817777 & 0,4 & $0 g_{7 / 2}$ & -4.791740 & -0.03287710 \\
\hline 4 & $1,-4$ & $1 f_{7 / 2}$ & -4.749130 & -0.01843870 & 0,5 & $0 h_{9 / 2}$ & -4.799920 & -0.05156860 \\
\hline 5 & $1,-5$ & $1 g_{9 / 2}$ & -4.791740 & -0.03287710 & 0,6 & & -4.791590 & -0.07461340 \\
\hline 6 & $1,-6$ & $1 h_{11 / 2}$ & -4.799920 & -0.05156860 & 0,7 & & -4.772990 & -0.102140 \\
\hline 7 & $1,-7$ & & -4.791590 & -0.07461340 & 0,8 & & -4.746630 & -0.134308 \\
\hline 8 & $1,-8$ & & -4.772990 & -0.102140 & 0,9 & & -4.713610 & -0.171314 \\
\hline 9 & $1,-9$ & & -4.746630 & -0.134308 & 0,10 & & -4.674380 & -0.213399 \\
\hline 10 & $1,-10$ & & -4.713610 & -0.171314 & 0,11 & & -4.629040 & -0.260854 \\
\hline 15 & $1,-15$ & & -4.454290 & -0.439464 & 0,16 & & -4.300520 & -0.594696 \\
\hline 20 & $1,-20$ & & -3.993200 & -0.903412 & 0,21 & & -3.708850 & -1.188370 \\
\hline 25 & $1,-25$ & & -2.938620 & -1.959250 & 0,24 & & -2.938620 & -1.959250 \\
\hline 1 & $2,-1$ & $2 s_{1 / 2}$ & -3.917958 & -0.00204188 & 1,2 & $1 d_{3 / 2}$ & -4.749130 & -0.01843870 \\
\hline 2 & $2,-2$ & $2 p_{3 / 2}$ & - & - & 1,3 & $1 f_{5 / 2}$ & -4.791740 & -0.03287710 \\
\hline 3 & $2,-3$ & $2 d_{5 / 2}$ & -3.917958 & -0.00204188 & 1,4 & $1 g_{7 / 2}$ & -4.799920 & -0.05156860 \\
\hline 4 & $2,-4$ & $2 f_{7 / 2}$ & -4.603587 & -0.00817777 & 1,5 & $1 h_{9 / 2}$ & -4.791590 & -0.07461340 \\
\hline 5 & $2,-5$ & $2 g_{9 / 2}$ & -4.749130 & -0.01843870 & 1,6 & & -4.772990 & -0.102140 \\
\hline 6 & $2,-6$ & $2 h_{11 / 2}$ & -4.791740 & -0.03287710 & 1,7 & & -4.746630 & -0.134308 \\
\hline 7 & $2,-7$ & & -4.799920 & -0.05156860 & 1,8 & & -4.713610 & -0.171314 \\
\hline 8 & $2,-8$ & & -4.791590 & -0.07461340 & 1,9 & & -4.674380 & -0.213399 \\
\hline 9 & $2,-9$ & & -4.772990 & -0.102140 & 1,10 & & -4.629040 & -0.260854 \\
\hline 10 & $2,-10$ & & -4.746630 & -0.134308 & 1,11 & & -4.577470 & -0.314039 \\
\hline 15 & $2,-15$ & & -4.519370 & -0.373394 & 2,16 & & -4.209880 & -0.685887 \\
\hline 20 & $2,-20$ & & -4.108150 & -0.788067 & 2,21 & & -3.525210 & -1.372260 \\
\hline 25 & $2,-25$ & & -3.291060 & -1.606630 & 2,23 & & -2.938620 & -1.95925 \\
\hline 26 & $2,-26$ & & -2.938620 & -1.959250 & & & & \\
\hline
\end{tabular}

